\title{
A Decomposition Algorithm for the Oriented Adjacency Graph of the Triangulations of a Bordered Surface with Marked Points
}

\author{
Weiwen $\mathrm{Gu}$ \\ Department of Mathematics \\ Michigan State University, East Lansing, USA \\ guweiwen@msu.edu
}

Submitted: Jul 13, 2010; Accepted: Apr 12, 2011; Published: Apr 21, 2011

Mathematics Subject Classification: $05 \mathrm{C} 88$

\begin{abstract}
In this paper we consider an oriented version of adjacency graphs of triangulations of bordered surfaces with marked points. We develop an algorithm that determines whether a given oriented graph is an oriented adjacency graph of a triangulation. If a given oriented graph corresponds to many triangulations, our algorithm finds all of them. As a corollary we find out that there are only finitely many oriented connected graphs with non-unique associated triangulations. We also discuss a new algorithm which determines whether a given quiver is of finite mutation type. This algorithm is linear in the number of nodes and is more effective than the previously known one (see [1]).
\end{abstract}

\section{Contents}

1 Introduction $\quad 2$

2 Definitions $\quad 5$

3 Simplification $\quad 8$

3.1 Nodes of Degree Eight . . . . . . . . . . . . . . . . . . 8

3.2 Nodes of Degree Seven . . . . . . . . . . . . . . . . . . . . . . . 9

3.3 Nodes of Degree Six . . . . . . . . . . . . . . . . . . . 10

3.4 Nodes of Degree Five . . . . . . . . . . . . . . . . . . . . 12 
4 Nodes of Degree Four 12

4.1 Four outward edges or four inward edges. . . . . . . . . . . . . . . . 13

4.2 Three outward edges and one inward edge, or three inward edges and one outward edge . . . . . . . . . . . . . . . . . 13

4.3 Two outward edges and two inward edges . . . . . . . . . . . . . 16

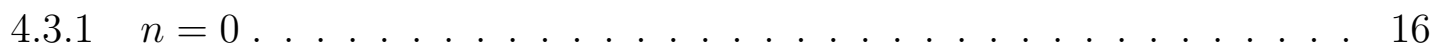

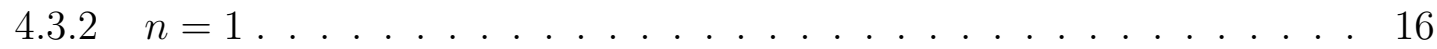

$4.3 .3 \quad n=2 \ldots \ldots \ldots \ldots \ldots \ldots \ldots \ldots \ldots$

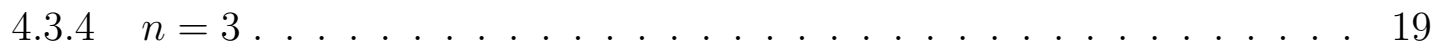

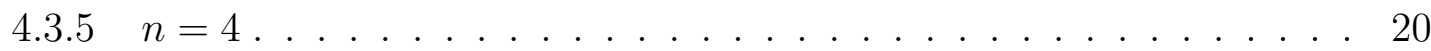

5 Distinguishing the Neighborhoods when $n=4 \quad 25$

5.1 Node $\mathbf{1}$ is Connected to Nodes $\mathbf{2}$ and $\mathbf{3}$. . . . . . . . . . . . . . 25

5.2 Node $\mathbf{1}$ is Connected to Node $\mathbf{2}$ but Disconnected from Node $\mathbf{3}$. . . . . . . 30

5.3 Node $\mathbf{1}$ is Disconnected from Nodes $2,3 \ldots \ldots 33$

6 Simplification on Nodes of Degree Three 34

6.1 All edges have the same direction. . . . . . . . . . . . . . . . . 34

6.2 Two outward edges and one inward edge $\ldots \ldots \ldots \ldots \ldots$

6.3 Determine the Decomposition . . . . . . . . . . . . . . . . . 41

$\begin{array}{lr}\text { References } & 45\end{array}$

\section{Introduction}

In this paper we consider the properties of triangulations of surfaces with marked points. We start with the following definitions from [2]:

Definition 1. Let $S$ be a connected oriented 2-dimensional Riemann surface with boundary. Fix a non-empty finite set $M$ of marked points in the closure of $S$ such that every connected component of the boundary has at least one marked point. We call $(S, M)$ a bordered surface with marked points if $(S, M)$ is none of the following:

- a sphere with one or two marked points in the interior of $S$.

- a disk with one marked point on the boundary, no more than one marked point in the interior.

- a disk with two marked points on the boundary, no marked point in the interior.

- a triangle with no marked points in the interior.

Definition 2. A (simple) arc $\gamma$ in $(S, M)$ is a curve in $S$ such that:

- the endpoints of $\gamma$ are marked points in $M$; 
- $\gamma$ does not intersect itself, except that its endpoints may coincide;

- except for the endpoints, $\gamma$ is disjoint from $M$ and from the boundary of $S$;

- $\gamma$ is not contractible into $M$ or onto the boundary of $S$.

Definition 3. A maximal collection of distinct pairwise arcs that do not intersect in the interior of $S$ is called an ideal triangulation. The arcs of a triangulation cut the surface $S$ into ideal triangles. The three sides of an ideal triangle do not have to be distinct, i.e., we allow self-folded triangles. We also allow for a possibility that two triangles share more than one side.

Definition 4. A quiver is defined as a finite oriented multi-graph without loops and 2-cycles.

Triangulations of surfaces provide a basic tool for study of surface geometry and topology. An important reference for us is [2] where the authors construct a cluster algebra associated with triangulations of a bordered surface with marked points. Moreover, they describe a distinguishing combinatorial property of such cluster algebra. Namely, exchange quiver of such cluster algebra is block decomposable. An exchange quiver is an oriented adjacency graph derived from the signed adjacency matrix associated to an ideal triangulation, defined as follows:

Definition 5. We associate to each ideal triangulation $T$ the (generalized) signed adjacency matrix $B=B(T)$ that reflects the combinatorics of $T$. The rows and columns of $B(T)$ are naturally labeled by the $\operatorname{arcs}$ in $T$. For notational convenience, we arbitrarily label these arcs by the numbers $1, \ldots, n$, so that the rows and columns of $B(T)$ are numbered from 1 to $n$ as customary, with the understanding that this numbering of rows and columns is temporary rather than intrinsic. For an arc (labeled) $i$, let $\pi_{T}(i)$ denote (the label of) the arc defined as follows: if there is a self-folded ideal triangle in $T$ folded along $i$, then $\pi_{T}(i)$ is its remaining side (the enclosing loop); if there is no such triangle, set $\pi_{T}(i)=i$. For each ideal triangle $\triangle$ in $\mathrm{T}$ which is not self-folded, define the $n \times n$ integer matrix $B^{\triangle}=\left(b_{i j}^{\triangle}\right)$ by settings:

$$
b_{i j}^{\triangle}= \begin{cases}1 & \text { if } \triangle \text { has sides labeled } \pi_{T}(i) \text { and } \pi_{T}(j) \\ & \text { with } \pi_{T}(j) \text { following } \pi_{T}(i) \text { in the clockwise order; } \\ -1 & \text { if the same holds, with the counter-clockwise order; } \\ 0 & \text { otherwise. }\end{cases}
$$

The matrix $B=B(T)=\left(b_{i j}\right)$ is then defined by

$$
B=\sum_{\triangle} B^{\triangle}
$$

The sum is taken over all ideal triangles $\triangle$ in $T$ which are not self-folded. The $n \times n$ matrix $B$ is skew-symmetric, and all its entries $b_{i j}$ are equal to $0,1,-1,2$, or -2 . 
Definition 6. Let $G$ be a quiver, $B(G)=\left(b_{i j}\right)$ is the skew-symmetric matrix whose rows and columns are labeled by the vertices of $G$, and whose entry $b_{i j}$ is equal to the number of edges going from $i$ to $j$ minus the number of edges going from $j$ to $i$.

Definition 7. Suppose $B$ is a signed adjacency matrix associated to an ideal triangulation of a bordered surface with marked points $(S, M)$, and $G$ is a quiver. If $B(G)=B$, we say $G$ is the oriented adjacency graph associated to $(S, M)$.

The notion of Block decomposition (see Definition 8) plays an important role in determining the mutation class of a quiver. In [2], the authors prove that the mutation class of an adjacency matrix associated to a triangulation of a bordered surface with marked points is finite (Corollary 12.2 in [2]). It is also proved in [2] that an integer matrix $B$ is an adjacency matrix of an ideal triangulation of a bordered surface with marked points if and only if $B=B(G)$ for some block-decomposable graph $G$.

In this paper, we provide a combinatorial algorithm that determines if a given graph is block-decomposable. Moreover, if a graph $G$ is block-decomposable, the algorithm can also find all possible bordered surfaces with marked points associated with $G$.

For a given graph $G$, we start the algorithm by examining the nodes of largest degree. Note that by construction (see Definition 8), the degree of any node of a block-decomposable graph does not exceed eight. We examine the nodes of degree eight one by one, and check the neighborhoods (see Definition 9) of the examined node, denoted by $o$. The set of decomposable neighborhoods of $o$ we need to check (we denoted it by $\mathcal{S}_{o}$, see remark 3 ) is finite. If $\mathcal{S}_{o}$ is empty, the graph $G$ is indecomposable and we terminate the algorithm. If $o$ is contained in a neighborhood $N \in \mathcal{S}_{o}$, we simplify $N$ in the following way: replace $N$ by a simpler neighborhood so that the degree of $o$ decreases. We prove that all replacements are consistent in the following sense: the original graph is block-decomposable if and only if the new graph is. After the nodes of degree eight are exhausted, we proceed in similar way to the nodes of degree seven, then, six, five and four. In each step, it is necessary to examine if $S_{o}$ of any node $o$ is non-empty. It is possible that after a few steps of simplification, we obtain several connected components. The same algorithm can be applied to each component. Eventually the graph is reduced to one with nodes of degree at most three. The decomposable neighborhoods of nodes of degree 3 are listed in Section 6 . Finally, Theorem 1 gives a criterion that determines if a graph that contains only nodes of degree at most three is decomposable.

Theorem 1. Assume that every node in $G$ has degree less than or equal to 3. If each of the nodes of degree three has a neighborhood as in one of the pictures listed in Figure 74 (up to the change of orientations of all edges), then $G$ is decomposable. Otherwise, $G$ is indecomposable.

Furthermore, the algorithm also provides a list of connected neighborhoods that are associated to non-unique triangulations, see the following theorem:

Theorem 2. If $G$ is a connected decomposable graph, it can only be one of the graphs from Figure 78, up to the change of orientations of all edges. Moreover, $G$ has finitely many possible decompositions. 

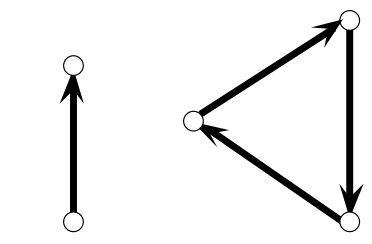

I:Spike
II:Triangle

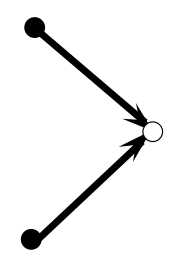

IIIa:Infork
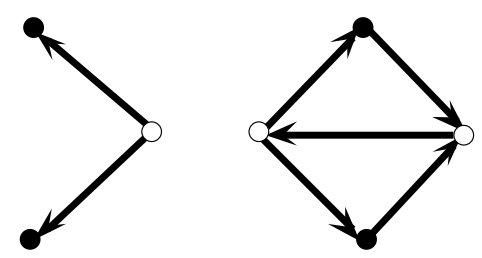

IV:Diamond

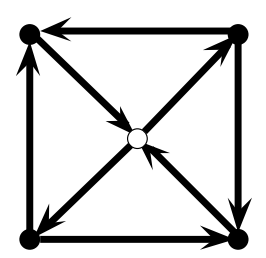

$\mathrm{V}:$ Square

Table 1: Blocks

The algorithm also helps to retrieve the triangulations and surfaces that a decomposable graph $G$ is associated with. We keep track of all neighborhoods that are simplified. After all nodes of degree higher than three are exhausted, we decompose the graph into blocks. Each elementary block is uniquely associated to a triangulation of a piece of surface. Gluing elementary blocks corresponds to a gluing of associated triangulated pieces of surfaces along arcs of triangulations (see [2]). Therefore, given a block decomposition, we can recover a triangulated bounded surface associated with the given quiver.

\section{Definitions}

For convenience, an edge directed from node $x$ to $y$ will be denoted by $\overrightarrow{x y}$; if an edge connects node $x$ and $y$, but the orientation is unknown or irrelevant, we denote the edge by $\overline{x y}$.

Definition 8. A block is a directed graph that is isomorphic to one of the graphs shown in Table 1. They are categorized as one of the following: type I (spike), II (triangle), IIIa (infork), IIIb (outfork), IV (diamond), and V (square). The nodes marked by unfilled circles are called outlets or white nodes. The nodes marked by filled circles are called dead ends or black nodes. A directed graph $G$ is called block decomposable or simply decomposable if it can be obtained from disjoint blocks as a result of the following gluing rules: (See [2] for definition.)

1. Two white nodes of two different blocks can be identified. As a result, the graph becomes a union of two parts, and the common node becomes black. A white node can not be identified with another node of the same block, see Figures 2.

2. A black node can not be identified with any other node.

3. If an edge $a:=\overrightarrow{x y}$ with two white nodes $x, y$ is glued to another edge $b:=\overrightarrow{p q}$ with two white nodes $p, q$ such that $x$ is glued to $p$ and $y$ is glued to $q$, then a multi-edge is formed, and the nodes $x=p, y=q$ become black. (Figure 1)

4. If an edge $a:=\overrightarrow{x y}$ with two white nodes $x, y$ is glued to another edge $b=\overrightarrow{q p}$ such that $x$ is glued to $p$ and $y$ is glued to $q$, then both edges are removed after gluing, 


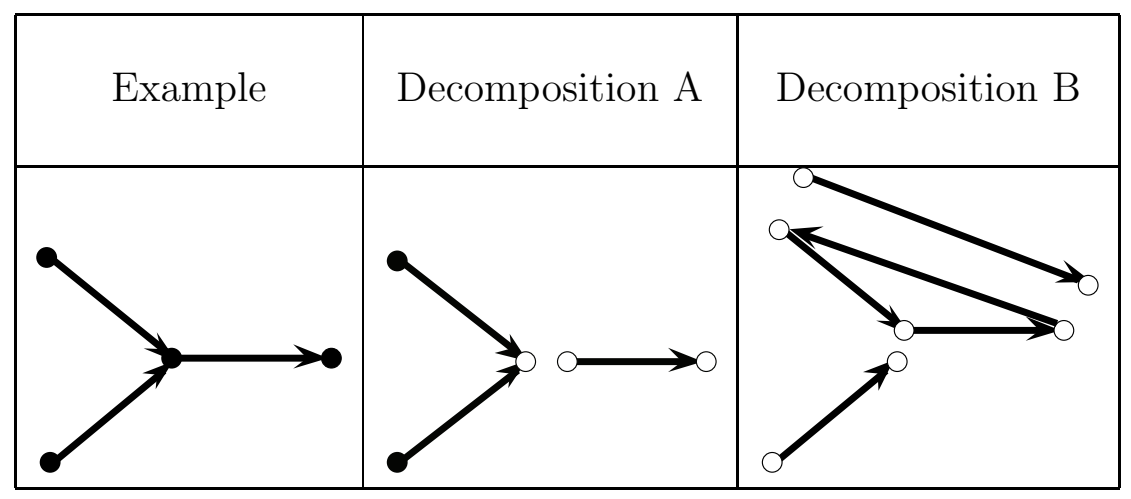

Table 2: A quiver and its two decompositions

and the nodes $x=p, y=q$ become black. We say that edges annihilate each other (Figure 2).

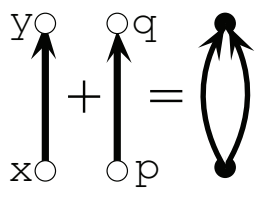

Figure 1

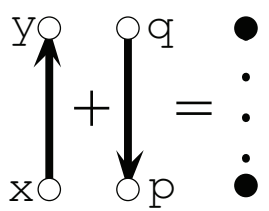

Figure 2

For example, the example quiver in Table 2 can be constructed from an infork (IIIa) and a spike (I) as in decomposition A, or from a spike (I), a triangle $(\mathbf{I I})$ and another spike(I) as in decomposition B.

Remark 1. By design, a block-decomposable graph has no loop and all edge multiplicities are 1 or 2 . Therefore, a block-decomposable graph is a quiver.

Remark 2. Note that, the color of a vertex is not specified in the original graph, and is determined only for vertices of blocks of a specified block decomposition. (There might be several ways to decompose a graph. Hence, a vertex may have different colors in different decompositions, see Figure 2.)

We will assume in the following discussion that $G$ is a finite oriented multi-graph without loops and 2-cycles.

Proposition 1. A graph $G$ without isolated nodes is decomposable if and only if every disjoint connected component is decomposable.

Proof. It suffices to show that annihilating an edge in a connected graph generates a connected graph. Since we can only annihilate edges in a spike, triangle or diamond block, before an edge is annihilated, both of its endpoints must be white. Denote these two endpoints by $x, y$. 
- Suppose $x, y$ are endpoints of a spike. Notice that the original graph must be a single spike. If we annihilate the edge by gluing a triangle, $x, y$ will be connected via the third node of the triangle. If we annihilate the edge by gluing a diamond, $x, y$ will be connected via the remaining nodes of the diamond. If we annihilate it by gluing a spike, the new graph will consist of only two nodes and no edge, a contradiction.

- Suppose $x, y$ are endpoints of a triangle. If we annihilate the edge $\overline{x y}$ by gluing a spike or diamond, the remaining two edges of the triangle can not be annihilated, and $x, y$ will still be connected via the third node of the triangular block. If we glue another triangle, there are two cases: In the first case, we can annihilate only one edge, namely $\overline{x y}$, and then $x, y$ will still be connected via the third node. In the second case, we can also annihilate the whole triangle when all three nodes are white. In this case, the original graph is a single triangular block and the new graph consists of three nodes. This is again a contradiction.

- Suppose $x, y$ are endpoints of a diamond. Since none of the boundary edges can be annihilated, after gluing a spike or triangle or another diamond to the edge $\overline{x y}, x, y$ will still be connected.

According to the previous proposition, if $G$ is decomposable, we may break connectivity of a graph in only two trivial cases. In either case, the resulting graph contains isolated nodes. On the other hand, in a decomposable graph, isolated nodes can only be obtained in the above manner. Therefore, we can assume from now on that the graph is connected.

The following definition is needed in our algorithm:

Definition 9. Suppose $N$ is a subquiver of $G$ with all its nodes colored white or black. If there exists another quiver $M$ with all its nodes colored white or black, such that $G$ can be obtained by gluing $M$ to $N$ by the rules in Definition 8, we say $N$ is a colored subquiver of $G$. A neighborhood of $o$ is a colored subquiver of $G$ that contains node $o$. We say a colored subquiver $N$ of $G$ is decomposable if there exists another block-decomposable graph $\widetilde{G}$ that contains $N$ as a colored subquiver. A colored subquiver $N$ of $G$ is said to be indecomposable if any graph that contains $N$ as a colored subquiver is indecomposable. We say a colored subquiver $N$ is decomposable as a subgraph if $N$ can be obtained by gluing elementary blocks according to the rules in Definition 8, and the color of nodes in $N$ resulted by gluing of blocks must be compatible with the original color of $N$.

Remark 3. First, note that if $G$ is obtained by gluing a colored subquiver to a neighborhood of $o$, no edge of the neighborhood can be annihilated. Secondly, for a given graph $G$ and a node $o$, the set of neighborhoods of $o$ in $G$, denoted by $\mathcal{N}_{o}$, forms a partially ordered set by inclusion. We define three subsets of $\mathcal{N}_{o}$ as follows:

- $\mathcal{I}_{o}$ is the set of all decomposable neighborhoods each of which contains all edges incident to $o$. 
- $\mathcal{D}_{o}$ is the set of all decomposable neighborhoods of $o$ each of which is decomposable as a subgraph.

- $\mathcal{S}_{o}=\left\{N \subset \mathcal{I}_{o} \cap \mathcal{D}_{o} \mid N\right.$ is minimal $\}$.

For example, consider the graph $G$ in the first picture of Table 3. In the first picture, note that as a quiver, $G$ does not have color on any node, hence the first picture is not considered as a neighborhood. Pictures $\mathbf{A}, \mathbf{B}, \mathbf{C}$ give some examples of neighborhoods of node $o$. The neighborhood in picture $\mathbf{A}$ belongs to $\mathcal{S}_{o}$. The neighborhood in picture $\mathbf{B}$ does not belong to $\mathcal{I}_{o}$. The neighborhood in picture $\mathbf{C}$ does not belong to $\mathcal{D}_{o}$. Note that although $\mathbf{C}$ as a graph can be obtained from gluing a spike and two triangles, the two nodes on the top will be black, which is incompatible with the coloring in picture $\mathbf{C}$.

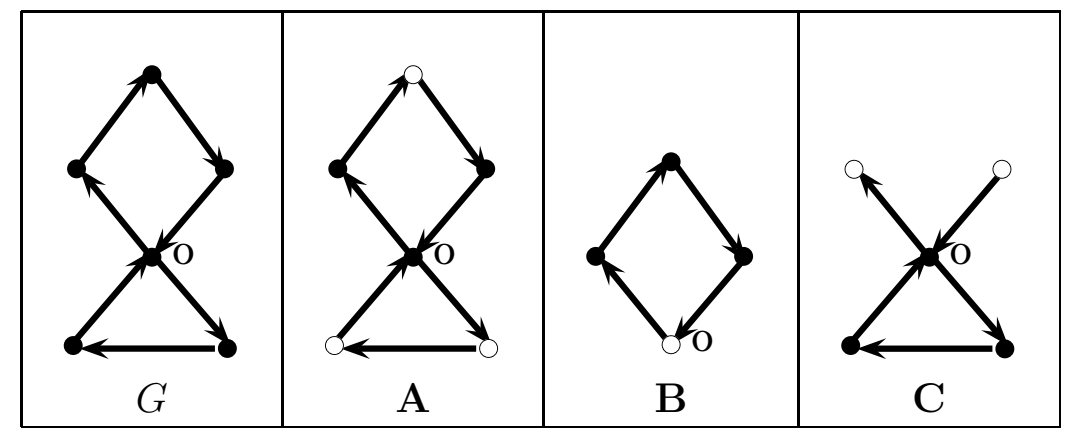

Table 3

For a given graph $G$ and a target node $o$, if $\mathcal{S}_{o}$ is empty, the graph is indecomposable. Remark 4. In our algorithm we only need to consider neighborhoods from $\mathcal{S}_{o}$,

\section{Simplification on Nodes of Degree Eight, Seven, Six and Five}

In this section we show when and how to replace the neighborhood of a certain node by a consistent one which decreases the degree of this node. As a result, the nodes of degree larger than four are consecutively eliminated. Notice that the highest degree of any node in a block is 4 . Hence the highest degree of any node in a decomposable graph $G$ does not exceed 8 .

\subsection{Nodes of Degree Eight}

A node $o$ of degree 8 in a decomposable graph $G$ can only be obtained by gluing a square with another square (see Figure 3). The result is a disjoint connected component. Otherwise, $G$ is indecomposable. 


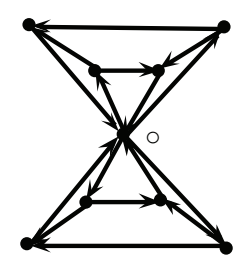

Figure 3: Node of degree 8

\subsection{Nodes of Degree Seven}

If $o$ is a node of degree 7 in a decomposable graph $G$, it must be obtained by gluing a diamond to a square, see Figure 4. The neighborhood is replaced by the one in Figure 5. The following lemma shows that this replacement is consistent.

Lemma 1. Suppose a neighborhood of the node o is as in Figure 5 and $\operatorname{deg}(o)=3$. If $G$ is decomposable, the neighborhood can only be decomposed into a triangle and a spike.

Proof. It is necessary to show that $b, c, d$ form a triangular block in the decomposition and that $a$ comes from a spike block.

Assume there exists a decomposition. We then claim that the block containing $b$ must be a triangle. Suppose that the claim is false, and consider the following cases:

1. Suppose that $b$ comes from a fork. Since both edges in a fork contain black endpoints, they can not be annihilated. Thus, the fork containing $b$ must also contain $a$ or $c$. However, the directions of $a$ and $c$ are not compatible with the directions of edges in any fork block. Therefore, $b$ can not be a part of a fork.

2. Suppose $b$ comes from a square block. Since at least one endpoint of any edge in a square block is black, none of the edges can be annihilated. Thus, the degree of any corner node is 3 , and the central node has degree at least 4 . Since the degree of node $o$ is 3, it can only be one of the corner node in the square. Moreover, since nodes $x$ and $p$ are not connected, they must both corner nodes on the same diagonal. Therefore, node $y$ must be the central node. Hence nodes $y$ and $p$ must be connected, a contradiction, and so $b$ can not come from a square.

3. Suppose that $b$ comes from a diamond. If the diamond does not contain $c$ or $d$, then it is necessary to glue $d$ and $c$ together. Since the only white nodes are the endpoints of the mid-edge, $b$ must be the mid-edge of the diamond. Suppose the diamond does not contain $c$. The edges $a, d$ must be both contained in the diamond since the degree of node $o$ is 3 . Hence nodes $x, p$ must be connected, which is a contradiction. Suppose the diamond does not contain $d$, then after gluing $d$ to node $o$, the degree of $o$ must be at least 4. This again leads to a contradiction. So the diamond must contain $d$ and $c$. The directions of $b, c$ suggest that both $b, c$ are in the upper or lower triangle of the diamond. This forces $d$ to be contained in the diamond. Notice that since the node $x$ is not connected with $p$, the other half of 
the diamond is annihilated. This is again a contradiction, so the diamond can not contain $c$. To conclude, $b$ is not contained in a diamond block.

4. Suppose $b$ comes from a spike, then $a, d$ must come from the same block. Thus, they form a fork, and so $c$ can not be attached. This proves the claim.

Now, the only option is that $b$ comes from a triangular block $\triangle_{1}$. If $a$ also comes from $\triangle_{1}$, the third edge in $\triangle_{1}$ should be annihilated by another edge, denoted by $e$. Moreover, both $e$ and $c$ must be obtained from the same block. Taking into account direction of edges, this block must be a triangle $\triangle_{2}$. Note that the third edge $\overline{p y}$ of $\triangle_{2}$ is annihilated by an edge $f$ incident to the node $y$, so $f$ and $d$ must come from the same block. Again, considering the directions of edges, this block must also be a triangle $\triangle_{3}$. Therefore, the third edge of $\triangle_{3}$ must be $a$, which contradicts the assumption that $a$ is an edge of block $\triangle_{1}$. Hence $a$ is not contained in $\triangle_{1}$ and this triangle is formed by $b, c, d$, which forces $a$ to be a spike block.

Remark 5. After the original neighborhood is replaced by the one in Figure 5, assume the new graph is not decomposable. This means that if the lower triangle and spike described in Lemma 1 are removed, the rest is not decomposable. Therefore, in the original graph, after the original neighborhood of $o$ is removed, the graph is indecomposable. However, in this case, the neighborhood of $o$ can only be obtained from gluing a square and a diamond. Hence the original graph is non-decomposable. This proves that the replacement is consistent. Moreover, all decompositions of the original graph are in 1-1 correspondence with decompositions of the new one.
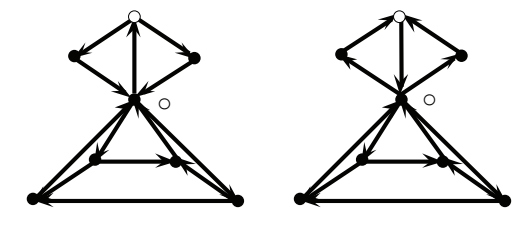

Figure 4: Node of Degree 7

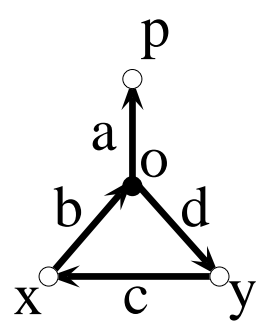

Figure 5

\subsection{Nodes of Degree Six}

If $o$ is a node in $G$ of degree 6, there are three cases:

1. One possible neighborhood in $\mathcal{S}_{o}$ comes from a triangle and a square block. (Figure 6 ) Then replace it by the one in Figure 7. Lemma 1 shows that this replacement is consistent.

2. The second possible neighborhood in $\mathcal{S}_{o}$ comes from a fork block (infork or outfork) and a square block. (Figure 8). Then the neighborhood is a disjoint connected component, since otherwise the graph is indecomposable. 


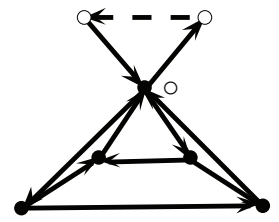

Figure 6

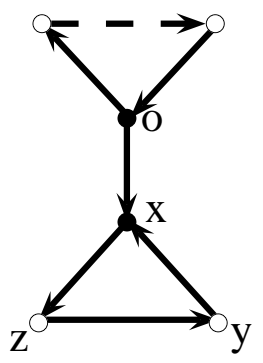

Figure 7

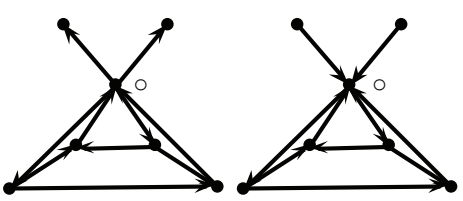

Figure 8

3. The third possible neighborhood in $\mathcal{S}_{o}$ comes from two diamonds, as illustrated in Figure 9. Note that in Figure 9A, if node $p$ and node $q$ are glued together, the result, if decomposable, is a disjoint connected component, see Figure 10A. Otherwise, $G$ is indecomposable. If $p, q$ are not glued together, the neighborhood is then replaced by the graph in Figure 11. Lemma 1 shows that this replacement is consistent. On the other hand, gluing nodes $p, q$ together in Figure 9B, the mid-edges are annihilated, as seen in Figure 10B. Hence, the degree of $o$ must be 4 . This contradicts the fact that node $o$ has degree 6. In this case, the neighborhood is replaced by Figure 11.

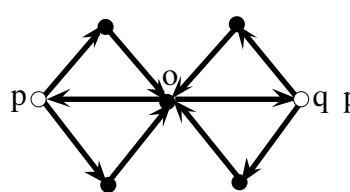

A

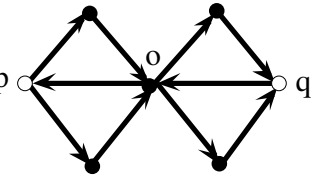

$\mathrm{B}$

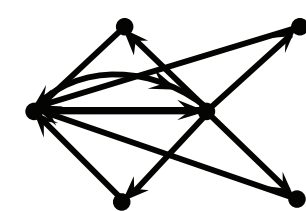

A

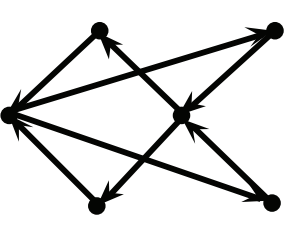

B

Figure 9

Figure 10

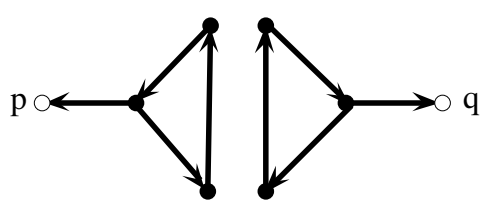

Figure 11

Corollary 1. Suppose a neighborhood of o is given as in Figure 7 and $\operatorname{deg}(x)=\operatorname{deg}(o)=3$. Then this neighborhood can only be decomposed as a triangle plus a spike plus a triangle.

Proof. By Lemma 1, the lower part of Figure 7 can only be obtained from gluing a spike and a triangle. For the upper part, the two edges incident to $o$ must come from the same block. Judging by their directions, the block can only be a triangle. Note that the third edge of this triangle may be annihilated, as indicated by a dashed line in Figure 7 .

Remark 6. By an argument similar to the one in remark 5, this replacement is consistent. 


\subsection{Nodes of Degree Five}

If node $o$ has degree 5 , there are three cases:

1. The first possible neighborhood in $\mathcal{S}_{o}$ comes from a spike and a square, see Figure 12. In this case, we replace it with the neighborhood in Figure 5. According to Lemma 1, the replacement is consistent.

2. The second possible neighborhood in $\mathcal{S}_{o}$ comes from a fork and a diamond, see Figure 13. Note that the direction of the fork and the diamond can change, so there are 4 subcases. In all of these cases replace the neighborhoods by the one in Figure 15. The replacement is consistent due to Lemma 1 and remark 5.

3. The third possible neighborhood in $\mathcal{S}_{o}$ comes from a triangle and a diamond, see Figure 14. In similarity with case 2 , note that the orientation of both the triangle and the diamond can also be reversed, and there are 4 possible neighborhoods in this case. Up to a reversion of directions, the neighborhood is replaced by the one in Figure 16. Lemma 1 and Corollary 1 ensure that this replacement is consistent.

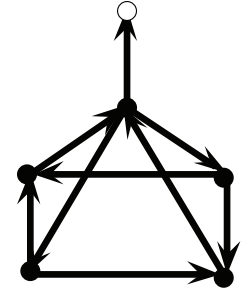

Figure 12

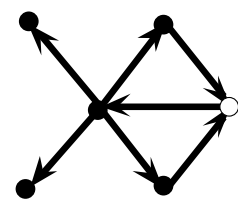

Figure 13

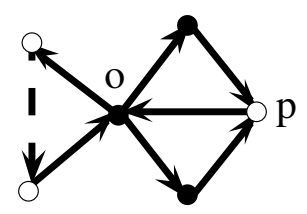

Figure 14

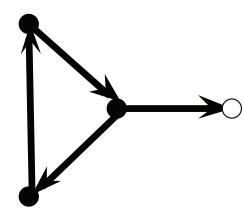

Figure 15

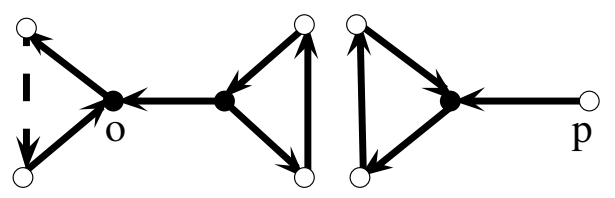

Figure 16

\section{Simplification on Nodes of Degree Four}

After the simplifications in the previous section are done, assume now that all nodes in graph $G$ have degrees at most four. In this section we shall denote the node in consideration by $o$, and the nodes connected to it are called boundary nodes. Note that taking into account the directions of edges incident to $o$, we can distinguish the following three cases:

A: 4 outward edges, or 4 inward edges.

B: 3 outward edges and 1 inward edge, or 3 inward edges and 1 outward edge. 
C: 2 outward edges and 2 inward edges.

We shall consider all the situations above case by case.

\subsection{Four outward edges or four inward edges.}

Without loss of generality, assume that there are four edges directed outwards. If the graph is decomposable, $\mathcal{S}_{o}$ consists of only one neighborhood, and the neighborhood is obtained by the gluing of two forks as in Figure 17.

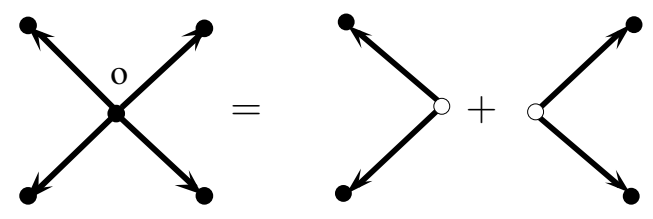

Figure 17

\subsection{Three outward edges and one inward edge, or three inward edges and one outward edge}

Without loss of generality, assume that $o$ is incident to three outward edges and one inward edge.

Assume that there is only one node, distinct from $o$ and incident to the incoming edge, that has degree at least two. Denote this node by $p$ :

1. The inward edge can not be obtained from a fork. To show this, we use contradiction. Suppose the edge is contained in a fork block, so $o$ must be the white node in this block. Therefore, the other inward edge must be incident to $o$ and can not be annihilated. This contradicts the fact that there is only one inward edge incident to $o$. Note that this argument is still true even if $p$ has degree one.

2. Suppose the inward edge comes from a square. Since the degree of $o$ is four, it must be the center of the square and all four edges are contained in the same square. This is impossible since none of the edges in a square can be annihilated and the central node of a square is incident to at least two inward edges and two outward edges.

3. Assume the inward edge is a part of a triangular block. Suppose this triangle does not contain any of the remaining three outward edges. Then the other edge of the triangle which is incident to $o$ is annihilated by another edge, denoted by $e$. In this case, $e$ and the remaining three outward edges must come from the same block. It can only be a square with central node $o$. On the other hand, $o$ is incident to three outward edges and one inward edge, giving us a contradiction. Therefore, the triangle must contain one of the outward edges, which forces the remaining two outward edges to be in the same block. This block must be a fork, see Figure 18. 
4. Assume that the inward edge comes from a diamond. Node $o$ must be a white node in the diamond. Judging by the directions of the remaining edges, two of them must be boundary edges of the same diamond, see Figure 19.

5. Suppose that the inward edge comes from a spike, then the remaining three outward edges come from the same block. However there is no block that contains three outward edges incident to the same node. Hence in this case, the graph is indecomposable.

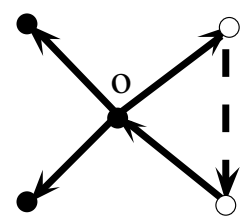

Figure 18

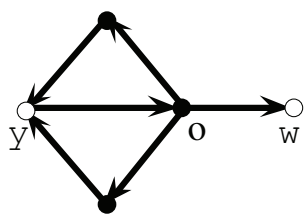

Figure 19

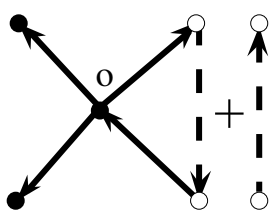

Figure 20

In Figure 18, replace the neighborhood with the one in Figure 7. According to Corollary 1 and remark 6, this replacement is consistent. Denote the new graph by $G^{\prime}$ and the original graph by $G$. $G^{\prime}$ has one less node of degree 4. For Figure 19, lemmas 2 and 3 show that it has a consistent replacement.

Lemma 2. If $y, w$ are not connected by an edge, Figure 21 has only one possible decomposition, which is shown in Figure 22.

Proof. Consider the edge $a$. We claim that it comes from a spike block. To justify the claim, we only need to rule out all other possibilities.

Suppose first that $a$ comes from a fork. Then the other edge of the same fork can not be annihilated since it has a black endpoint. Hence it must be edge $b$ or $c$. Assume it is $b$, then the degree of $b$ must be one, a contradiction.

Suppose now that $a$ comes from a square. Since the degree of $o$ is 4 , the node $o$ must be the center of the square, which means edges $b, c, f$ are contained in the same square block. This is a contradiction, since $o$ must be at least incident to two outward edges and two inward edges.

Suppose $a$ is contained in a diamond. The degree of node $o$ suggests that $o$ is a white node in the diamond block containing $a$. Since the boundary edges of a diamond can not

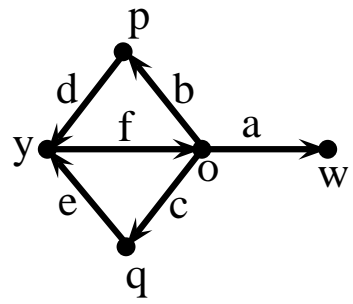

Figure 21

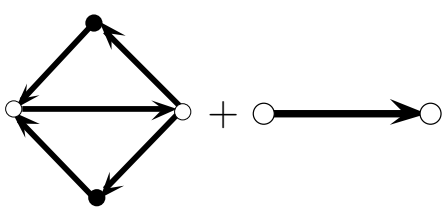

Figure 22

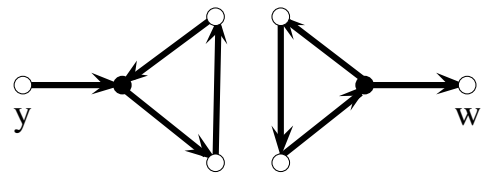

Figure 23 


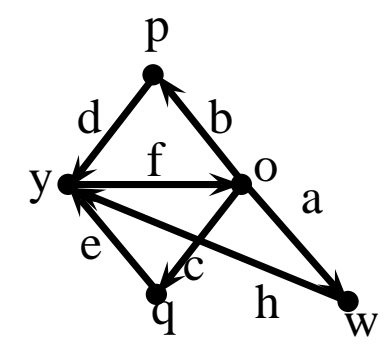

Figure 24

be annihilated, two of $a, b, c, f$ must be boundary edges. Judging by the directions, the boundary edges can only be $\{a, b\},\{a, c\}$ or $\{b, c\}$. If $\{a, b\}$ are two boundary edges, then $d$ must be contained in the same diamond. This means that the node $w$ must be connected to the node $y$, which contradicts our assumption. The situation is similar if $\{a, c\}$ are two boundary edges. If $\{b, c\}$ are two boundary edges of the diamond, $a$ is the mid-edge of the same diamond block. Therefore nodes $p, q$ must be connected with node $w$, and they must be black. However, edges $d, e$ are incident to them, again a contradiction.

Finally, suppose $a$ comes from a triangular block. If this triangle does not contain edge $f$, the other edge of the same triangle which is incident to $o$ must be annihilated by another edge, denoted by $h$. So $b, c, f, h$ come from the same block, which must be a square. However, none of the edges in a square can be annihilated, which contradicts the fact that $h$ is annihilated. If the triangle contains $f$, then $b, c$ must come from the same block, which must be a fork or a diamond. If it is the latter, the mid-edge must be annihilated. But $o$ is already a black node once the triangle and diamond are glued together, a contradiction. If it is a fork, the degree of $p$ must be one. This is again a contradiction.

To sum up, $a$ must be a single spike, and $b, c, f$ come from the same block. This forces the block to be a diamond.

Lemma 3. If $w$ is connected to $y$ in Figure 21, then the decomposable graph must have a disjoint connected component as shown in Figure 24.

Proof. According to the previous lemma, there are two possibilities. Either $b, c, d, e, f$ form a diamond and $a, h$ come from two spikes, or $a, b, d, f, h$ form a diamond and $e, c$ come from two spikes. In either case, the neighborhood is a disjoint connected component. Figure 24 illustrates the first case. To see the second case, one only needs to change the labeling of the edges in Figure 24.

Remark 7. The replacement of the graph in Figure 22 by the one in Figure 23 is consistent.

Assume now the node incident to the edge directed inwards has degree one.

1. Suppose that the inward edge comes from a spike. We show that the remaining three edges can not come from one block, and this contradicts decomposability. Indeed, there is no block that contains a node of degree 3 , that is incident to three outward edges. 


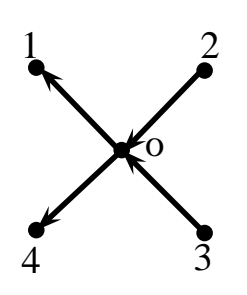

Figure 25

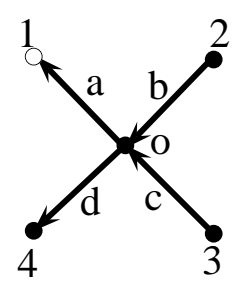

Figure 26

2. Suppose the inward edge comes from a fork. Since the degree of $o$ is four, $o$ must be the white node in the fork. Hence one of the remaining edges is contained in the same fork. However, their directions are inconsistent with a fork, giving a contradiction.

3. The inward edge can not be obtained from a diamond since every node in a diamond has degree at least 2 .

4. The same argument shows that the inward edge does not come from a square.

5. If the inward edge is obtained from a triangle, then by arguments as in the proof of Lemma 2, the triangle must contain one of the remaining outward edges. The only possible decomposition is shown in Figure 20. The dashed edge can only be annihilated by a spike, since otherwise the degree of the node will be greater than one. In this case, the neighborhood is a disjoint connected component.

\subsection{Two outward edges and two inward edges}

Here we will distinguish cases by the number of boundary nodes of degree at least 2 . Denote the number of such nodes by $n$. For example, if $n=0$, it is a 4 -star.

\subsection{1 $n=0$}

The neighborhood consisting of all four edges incident to $o$ can be constructed from gluing two forks, as shown in Figure 25. Also, it can be constructed from gluing two triangles, each triangle with one edge annihilated. It must be a disjoint connected component, otherwise $G$ is indecomposable.

\subsection{2 $n=1$}

Without loss of generality, assume the node $\mathbf{1}$ incident to an outward edge has degree at least 2 (Figure 26). Then we have the following cases:

1. Edge $a$ does not come from a fork since the degrees of both nodes $\mathbf{1}$ and $o$ are at least 2 .

2. Suppose $a$ comes from a diamond. Since the degrees of nodes $2,3,4$ are all 1 , they can not be contained in the same diamond. So node $o$ is a white node of the diamond 


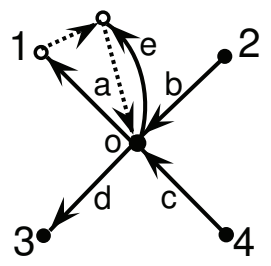

Figure 27

before attaching edge $b, c, d$. Hence at least one boundary edge in the diamond must be annihilated, which is impossible.

3. Suppose $a$ comes from a square. If $o$ is the central node of the square, edges $b, c, d$ must be contained in the same square. Hence the remaining 4 nodes must be corner nodes. Thus, they all have degree 3. This is a contradiction since only node $\mathbf{1}$ has degree more than one. So $o$ is a corner node of the square. But then the degree of node $o$ must be three, which contradicts the fact that the degree of $o$ is four.

4. If $a$ comes from a spike block, $b, c, d$ must come from the same block, which must be a diamond. Hence, edge $d$ is the mid-edge. However the degree of node $\mathbf{3}$ is 1 , which is impossible since no boundary edge in a diamond can be annihilated.

5. Assume that $a$ comes from a triangle $\triangle$. If the other edge of $\triangle$ incident to $o$ is not $b$ or $c$, that edge must be annihilated by another one denoted by $e$, as shown in Figure 27. Thus, $b, c, d, e$ come from the same block, which can only be a square. But the degrees of nodes $\mathbf{2 , 3 , 4}$ are all 1, which is impossible for nodes in a square block, so either $b$ or $c$ is contained in the same triangle. Assume that it is $b$. Notice that node $\mathbf{2}$ has degree 1 . So the edge in $\triangle$ that connects node $\mathbf{1}$ and $\mathbf{2}$ is annihilated by another edge, denoted by $f$. If $f$ comes from a spike, the degree of node $\mathbf{1}$ must be 1 after gluing, a contradiction. If $f$ comes from a triangle or a diamond, the degree of node $\mathbf{2}$ has degree at least 2 after gluing, also a contradiction.

To conclude, when $n=1$, the graph is indecomposable.

\subsection{3 $n=2$}

In this case, only two boundary nodes have degree at least 2 .

Case 1. Assume that the edges incident to the boundary nodes of degree at least 2 have the same direction. Without loss of generality we assume that both are directed outwards (nodes $\mathbf{1}$ and $\mathbf{4}$ in Figure 28 have degree at least 2.) First, suppose either $a$ or $d$ is a single spike. The remaining three edges must come from the same block, which can only be a diamond or a square. However, the degrees of nodes $\mathbf{2 , 3}$ are both 1 , which is impossible. Second, neither of the edges $a$ or $d$ can be obtained from a fork since both of its two endpoints have degree at least 2. Third, suppose $a$ comes from a diamond. Then $b, c$ must also be contained in the diamond. In this case, nodes $\mathbf{1}$ and $\mathbf{2}$ must be connected. This means the degree of node $\mathbf{2}$ is at least 2, which leads to a contradiction. 
Next, suppose $a$ or $d$ comes from a square, then all four edges must be contained in the same square. However, the degrees of node $\mathbf{2 , 3}$ are both one. This is again a contradiction. Last of all, assume $a, b$ come from the same triangular block and $c, d$ come from another triangular block. Since node 2 has degree 1, the third edge in the triangle containing edges $a, b$ is annihilated, as discussed in the case when $n=2$, this is a contradiction. So in this case, the graph is not decomposable. Case 2. Assume now that $o$ is connected to

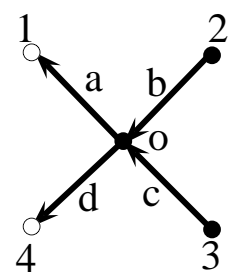

Figure 28

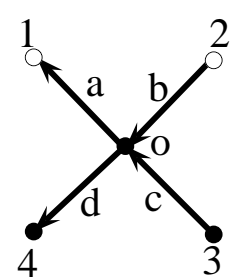

Figure 29

the boundary nodes of degree at least 2 by two edges. Denote the edge directed inwards by $a$ and the one directed outwards by $b$ (Figure 29).

1. For the same reason as in Case 1, neither $a$ nor $b$ comes from a spike block.

2. Neither $a$ nor $b$ comes from a fork since both endpoints have degrees at least 2 .

3. Suppose a comes from a diamond. Since the degree of $o$ is 4 , it must be a white node in the diamond. Since no boundary edge in a diamond can be annihilated, $b, c$ must be boundary edges in the same diamond. Then, nodes $\mathbf{1}$ and $\mathbf{3}$ must be connected. But degree of node $\mathbf{3}$ is 1 and the boundary edge can not be annihilated, which is a contradictions.

4. Suppose $a$ comes from a square block. Since degree of $o$ is 4 , it must be the central node of the square. Since none of the edges in a square can be annihilated, $a, b, c, d$ must all be contained in the same square. But the degrees of node $\mathbf{3 , 4}$ are one, a contradiction.

5. Assume $a$ comes from a triangle $\triangle$ that does not contain $b$ or $c$. The other edge in this triangle that is incident to $o$ must be annihilated by an edge denoted by $e$. Hence edges $b, c, d, e$ must be contained in a square block. However, none of the edges in a square block can be annihilated. Therefore, the triangle must contain either $b$ or $c$. Using similar arguments as in section 4.3.2, $c$ is not contained in $\triangle$, so $a, b$ are contained in $\triangle$. Then we replace the neighborhood consisting of all four edges incident to $o$ with the one in Figure 30. The replacement operation is consistent by Corollary 1. 


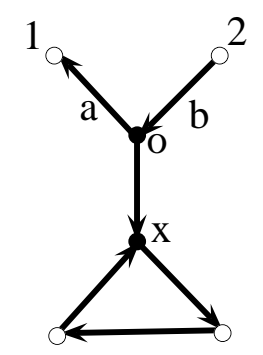

Figure 30: Case 2 replacement

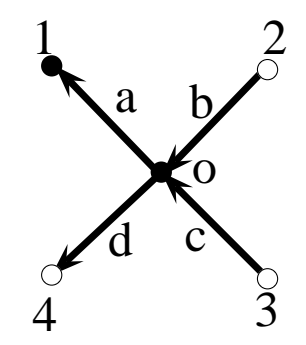

Figure 31

\subsection{4 $n=3$}

Without loss of generality, assume that the node $\mathbf{1}$ is incident to the edge directed outwards (denoted by $a$ ), and that it has degree one, see Figure 31.

1. Suppose that $a$ comes from a single spike. The remaining edges $b, c, d$ must come from the same block. The only possible situation is that they come from a diamond (Figure 32 ). Since $\operatorname{deg}(\mathbf{1})=1$, nodes $\mathbf{1 , 4}$ are not connected. We will show in Lemma 4 that this neighborhood consisting of the spike and the diamond can be replaced by the one in Figure 33. This replacement is consistent according to Lemma 1.

2. Suppose that a comes from a fork. Since $\operatorname{deg}(o)=4, o$ must be the white node in the fork. Then $d$ is also contained in the same fork. Hence, node 4 must have degree one. This contradicts the fact that the degree of node $\mathbf{4}$ is at least two, so $a$ does not come from a fork.

3. Assume a comes from a triangle. According to the argument in section 4.2, this triangle must contain edge $b$ or $c$ Assume that the triangle contains $a, b$. Since the degree of node $\mathbf{1}$ is one and the degree of node $\mathbf{2}$ is at least two, we obtain a contradiction by arguments from section 4.3.2.

4. Suppose that a comes from a diamond, then the degree of node $\mathbf{1}$ must be at least 2 , which is a contradiction.

5. Suppose that a comes from a square block. This block must also contain edges $b, c, d$ since none of the edges in a square can be annihilated. Moreover, $o$ is the 
central block of the square, which means node $\mathbf{1}$ must have degree 3 . This is a contradiction.

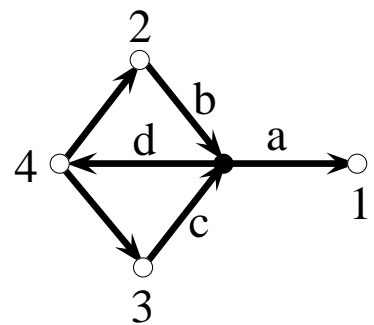

Figure 32

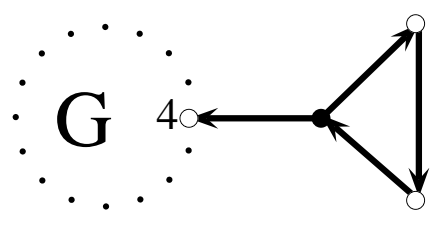

Figure 33: $G^{\prime}$

\subsection{5 $n=4$}

In this section, we assume all four boundary nodes have degree at least 2. (Figure 34). We focus our discussion on edge $a$. By the symmetry of the neighborhood, we can carry the same argument to any of the edges $b, c, d$.

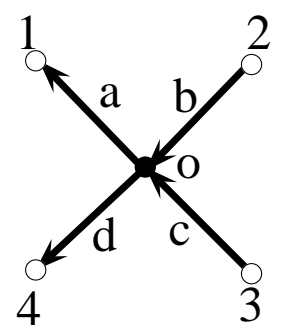

Figure 34

1. Edge $a$ does not come from a fork since both of its endpoints have degrees at least 2 .

2. Assume that a comes from a triangle. Similar to the argument in section 4.2 , the triangle must contain $b$ or $c$. Assume $b$ is contained in this triangle. Then $c$ and $d$ must come from the same block.

- If $c, d$ are contained in a diamond block, judging by their directions, one of edges $c, d$ (assume it is $d$ ) must be the mid-edge. Thus, besides $c$, there is another boundary edge incident to $o$ that comes from the same diamond. Hence, the degree of $o$ is at least 5, which contradicts our assumption.

- Assume $c, d$ come from a triangle, as shown in Figure 35. We replace the neighborhood in Figure 34 by the one in Figure 36. 


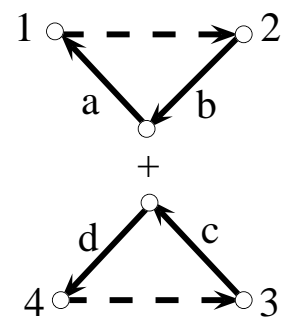

Figure 35

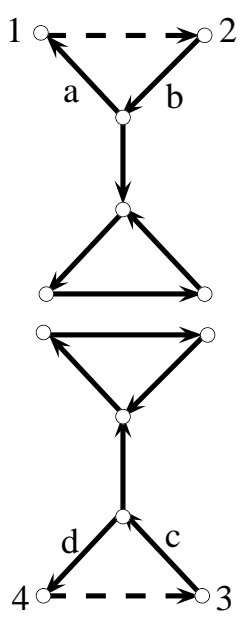

Figure 36

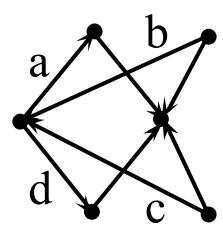

Figure 37

Remark 8. Notice that in order to perform a replacement, it is necessary to determine whether $a, b$ or $a, c$ are in the same triangle. This will be discussed later.

3. Suppose that a comes from a diamond. Since the degree of $o$ is 4 , it must be a white node of the diamond. Judging by the directions of edges, there are three cases.

- $a$ is the mid-edge and $b, c$ are the boundary edges. We get a neighborhood as shown in Figure 38. In this neighborhood, the degrees of nodes $\mathbf{2}$ and $\mathbf{3}$ are both 2 . We will discuss this case later in this section.

- $a, d$ are the boundary edges and $b$ or $c$ is the mid-edge. We get a neighborhood shown in Figure 39. In this neighborhood, the degrees of nodes $\mathbf{1}$ and $\mathbf{4}$ are both 2 .

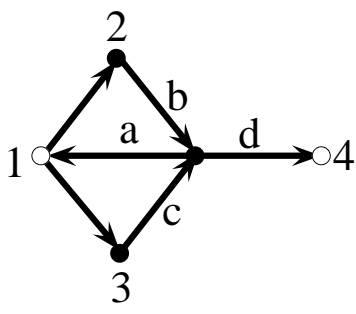

Figure 38

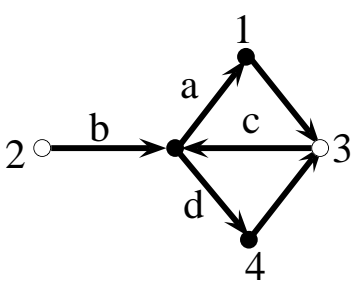

Figure 39

- $a, d$ are the boundary edges, and the mid-edge is annihilated by another edge $e$. So $b, c, e$ come from the same block. It must be a diamond with mid-edge $e$, see Figure 37. In this case, the neighborhood is a disjoint connected component.

4. If $a$ comes from a spike, $b, c, d$ must come from the same block. Hence, this block must be a diamond, see Figure 32. 
5. Suppose $a$ comes from a square, then $a, b, c, f$ must all be contained in the same square. Thus, node $o$ must have a neighborhood that comes from a square with $o$ as its central node. Since the degree of $o$ is 4 , the neighborhood is a disjoint connected component.

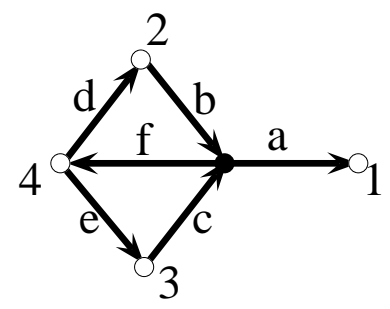

Figure 40

Note that Figures 38, 39, 32 represent the same neighborhood except for edge labeling. For the sake of convenience, we relabel the edges as in Figure 40. Note that the degree of node $\mathbf{1}$ is at least 2 . If node $\mathbf{1}$ is not connected to node $\mathbf{4}$, the only possible decomposition is the one shown in Figure 41 (see Lemma 4). We apply the replacement as in Figure 23. If nodes $\mathbf{1 , 4}$ are connected by an edge directed from 1 to 4 , Lemma 5 shows that there exists a decomposition in Figure 43. Thus, we can apply the replacement as in Figure 45. The following lemmas show that our choices of replacements for the neighborhood in Figure 40 are consistent.

Lemma 4. In Figure 40, assume 4 is neither connected to 1 nor coincides with 1. Assume further that nodes 1,2 and nodes 1,3 are disconnected. If the graph $G$ is decomposable, then the neighborhood in Figure 40 can be decomposed as in Figure 41.

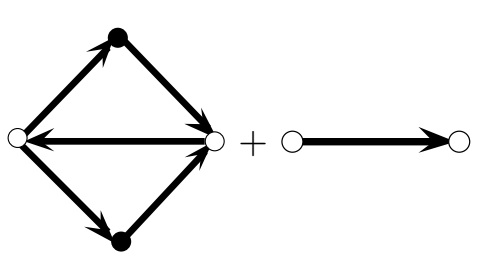

Figure 41

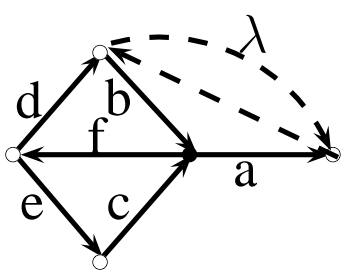

Figure 42

Proof. We have the following cases:

1. Suppose that $a$ comes from a fork. Since the degree of $o$ is $4, o$ must be the white node in the fork. Thus, $f$ is contained in the same fork. Then node 4 must have degree 1 , a contradiction. 
2. Suppose that a comes from a triangle, denoted by $\triangle$. Then there are two cases: Case 1: $\triangle$ contains neither $b$ nor $c$;

Case 2: $\triangle$ contains either $b$ or $c$

In case 1 , consider node $o$ in Figure 40. The other edge in $\triangle$ that is incident to $o$ is annihilated by another edge, denoted by $e$. Hence $b, c, f, e$ come from the same block, which can only be a square block. However, none of the edges in a square can be annihilated. Therefore, case 1 is impossible. In case 2 , assume that $\triangle$ contains $b$. The third edge in $\triangle$ is annihilated by another edge, denoted by $\lambda$ (see Figure 42 ). $\lambda$ and $d$ must come from the same block, which can only be a diamond or a triangle. If it is a diamond, $\lambda$ must be the mid-edge, so node 4 is black. However, edges $f, e$ need to be glued to $\mathbf{4}$, a contradiction, so both $b, \lambda$ belong to a triangle. Since 4 is not connected to $\mathbf{1}$, the edge $\overline{14}$ in this triangle must be annihilated by another edge $h$. So $h, f, e$ come from the same block, which must be a diamond with $h$ as its mid-edge. This means that nodes $o$ and $\mathbf{1}$ are connected by a boundary edge of this diamond. Thus, the degree of $o$ is at least 5, which contradicts the assumption that $\operatorname{deg}(o)=4$.

3. Suppose that a comes from a diamond. Since the degree of $o$ is 4 , it must be a white node in the diamond. Since the boundary edges can not be annihilated, and judging by the directions of the edges, there are only two possible cases:

- $a$ is the mid-edge and $b, c$ are two boundary edges of the diamond.

- $a, f$ are the boundary edges and one of $b, c$ is the mid-edge.

In either case, $\mathbf{1 , 2}$ must be connected by a boundary edge and it can not be annihilated, giving a contradiction.

4. Suppose that a comes from a square, then $a, b, c, f$ must all be contained in the same square. Thus, the neighborhood is the square. Moreover, nodes 1,2 must be connected, a contradiction.

5. Suppose edge $a$ comes from a spike. Then $b, c, f$ come from the same block, which forces the block to be a diamond, see Figure 41.

Lemma 5. In Figure 40, assume that 4 is connected to 1 by an edge directed from 1 to 4. If the graph is decomposable, nodes 1,2 and nodes 1,3 are disconnected. Then the degree of $\mathbf{4}$ is 4 and the degree of $\mathbf{1}$ is 2. In this case, there is a decomposition as in Figure 43. Also, it is possible to simplify the original graph $G$ to $G^{\prime}$ (Figure 45), and $G$ is decomposable if and only if $G^{\prime}$ is decomposable. If the degree of node 3 is 2 , there is an alternative decomposition as in Figure 44. In this case, $G$ is a disjoint connected component. 


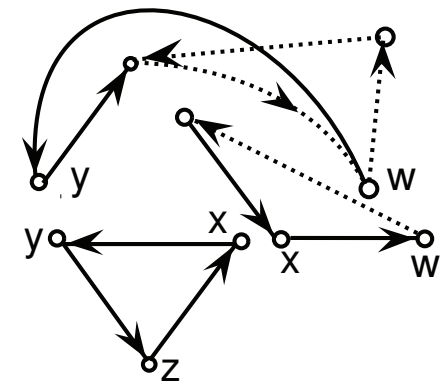

Figure 43

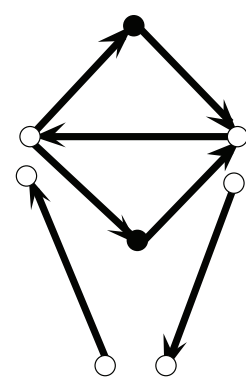

Figure 44

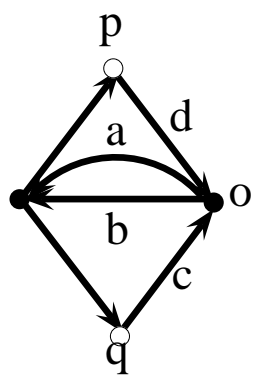

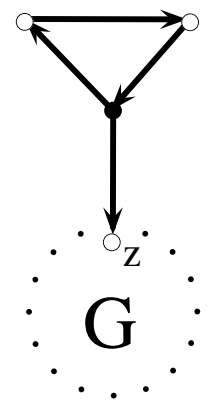

Figure 45: G'

Figure 46

Proof. The argument differs from the previous one only in the place where $a$ is assumed to come from a triangle. Notice that 4 is connected to $\mathbf{1}$. If $a, b$ comes from a triangular block $\triangle$, the edge $\overline{41}$ must come from another block. This block can be a triangle or a diamond. In this case, $e, f$ must both come from the other block, which can not be a diamond since this will force the degree of node 4 to be 5 . Recall that we already simplified all nodes of a decomposable graph so that the degree of any node does not exceed 4 . Thus, this block containing $e, f$ must be a triangle. (The corresponding decomposition is shown in Figure 43). In this case, if the degree of node $\mathbf{3}$ is at least 3, there is another edge incident to it. The neighborhood can be replaced by the one in Figure 45. It is trivial that if $G$ is decomposable, so is $G^{\prime}$. The converse statement follows from Lemma 1.

Remark 9. If node $\mathbf{4}$ coincides with node $\mathbf{1}$, we have a neighborhood as in Figure 46. In this case, we need to examine nodes $p, q$.

- If both nodes have degree two, then there are two possible decompositions. Namely, a diamond plus a spike or two triangles.

- If at least one of $p, q$ has degree more than two, then it must come from gluing two triangles.

If the node does not have either of the above two neighborhood, the graph is indecomposable. 


\section{$5 \quad$ Distinguishing the Neighborhoods when $n=4$}

In the previous section, we have discussed all cases when a node with degree 4 is formed. We also found necessary decompositions in all these cases. However, for a given graph $G$, when $n=4$, the question still remains when a node $o$ of degree 4 has a neighborhood contained in $\mathcal{S}_{o}$, and which neighborhood it has. For example, in the situation when the neighborhood may come from two triangles (see Figure 35), in order to choose proper replacement, we must determine whether $a, b$ or $a, c$ are in the same triangle. In this section, we focus on the case when $\operatorname{deg}(o)=4$ and $n=4$. We want to use only the information that can be directly derived from the graph:

- How is the node $o$ connected to the boundary nodes? We want to check the direction of the edges connecting node $o$ and its boundary nodes.

- How are the boundary nodes connected to each other? We want to check if, and how, some of the boundary nodes are connected to each other.

- If necessary, we want to check if there is any other node that is connected to the boundary nodes, and how they are connected.

First of all, we examine the neighborhoods of $o$ by checking how nodes $\mathbf{3 , 4}$ are connected to node $\mathbf{1}$.

\subsection{Node 1 is Connected to Nodes 2 and 3}

Assume that nodes $\mathbf{1 , 4}$ are connected by an edge denoted by $\lambda$ and nodes $\mathbf{1 , 3}$ are connected by an edge denoted by $\gamma$. Let us consider directions of $a, d, \lambda$ and $a, c, \gamma$. More exactly, we check if $\lambda$ is directed from node $\mathbf{1}$ to node $\mathbf{4}$ and if $\gamma$ is directed from node $\mathbf{1}$ to $\mathbf{3}$ (see Figure 47).

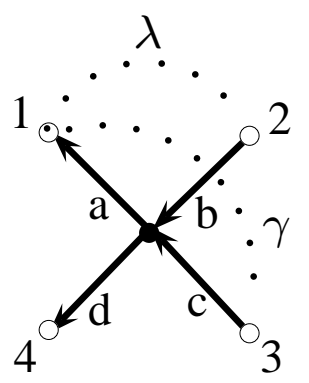

Figure 47

Suppose $\lambda$ is directed from node $\mathbf{2}$ to node $\mathbf{1}$ and $\gamma$ is directed from node $\mathbf{3}$ to $\mathbf{1}$, then neither $a, b, \lambda$ nor $a, c, \gamma$ come from a triangle. Assume $a$ comes from a spike, then $b, c, d$ come from the same block which must be a diamond. Hence, nodes $\mathbf{2 , 4}$ must be connected and node $\mathbf{2}$ is a black node before $\lambda$ is attached. In this case, node $\mathbf{1}$ must coincide with 
node 4. But the directions of $\lambda, \gamma$ are prescribed by the decomposition. Hence, the graph is indecomposable. If $a$ comes from a diamond, the diamond must also contain $b, c, \lambda, \gamma$. Again, their directions do not fit in a diamond block. To conclude, if $a, b, \lambda$ or $a, c, \gamma$ can not form a triangular block, the graph is indecomposable.

Suppose $a, b, \lambda$ have the same direction setup as a triangular block, and $a, c, \gamma$ do not. We claim that if the graph is decomposable, then $a, b, \lambda$ must come from a triangular block. Suppose the contrary. Notice that $a, c, \gamma$ do not come from a triangular block. Edge $a$ comes either from a spike or from a diamond. In the first case, $b, c, d$ come from the same block which must be a diamond, and node $\mathbf{2}$ is connected only to nodes $\mathbf{4}$ and $o$. Since node $\mathbf{2}$ is connected to $\mathbf{1}$, node $\mathbf{1}$ must coincide with node $\mathbf{4}$. But then the direction of $\gamma$ does not match the direction of the corresponding edge in a diamond. This is a contradiction. If $a$ comes from a diamond block, the block can contain $b, c, \lambda, \gamma$ or $b, d, \lambda$ or $c, d, \gamma$. But none of these cases has the directions that match with a diamond block. This again leads to a contradiction.

Suppose $\lambda=\overrightarrow{\mathbf{1 2}}$ and $\gamma=\overrightarrow{\mathbf{1 3}}$. If $a$ comes from a spike, then $\lambda, \gamma$ come from the same block. This block can be a fork or a diamond. But the former is impossible since the degree of node $\mathbf{2}$ is 2 . If it is the latter, $b, c$ must be boundary edges of this diamond. Thus, the mid-edge must connect node $\mathbf{1}$ and $o$. This forces node $\mathbf{1}$ to coincide with node 4, as shown in Figure 46. Suppose that a come from a diamond. There are two possibilities. Either $a, b, d$ come from the same diamond, or, $a, b, c$ come from the same diamond. If it is the former, node $\mathbf{1}$ is already black before $\gamma$ is glued, which is impossible. Suppose it is the latter. Notice that nodes $\mathbf{2 , 3}$ and $\mathbf{3 , 4}$ are disconnected unless node $\mathbf{1}$ coincide with node 4.

Lemma 6. Suppose there is an edge directed from node $\mathbf{1}$ to node $\boldsymbol{2}$ and an edge directed from node 1 to node 3. Furthermore, assume that nodes 2,3 and node 3,4 are disconnected, and that both nodes 3 and 4 have degree 2. If the graph $G$ is decomposable, then there is a decomposition of $G$ in which $a, b, c, \lambda, \gamma$ come from the same diamond.

Proof. Suppose that the conclusion of the lemma is false.

1. If a comes from a spike, then $b, c, d$ come from the same block which must be a diamond. Thus, node $\mathbf{3}$ is a black node of the diamond and $\gamma$ can not be attached, a contradiction.

2. If $a$ comes from a triangle, the block could contain either edges $b, \lambda$ or edges $c, \gamma$. In the former case, edges $c, d$ come from the same triangle $\triangle$. Since nodes $\mathbf{3 , 4}$ are disconnected the third edge of $\triangle$ is annihilated by another edge, denoted by $\tau$. Hence $\tau, \gamma$ come from the same triangle, and node $\mathbf{1}$ is connected to 4 . If $G$ is decomposable, the neighborhood is as in Figure 48. Moreover, the degree of node $\mathbf{1}$ is four and the degree of node $\mathbf{4}$ is two. Notice that the degree of node $\mathbf{2}$ is two, hence the neighborhood is a disjoint connected component. In this case, it can also be decomposed as a diamond containing $a, b, c, \lambda, \gamma$ plus two spikes. In the latter case, the triangle which contains $a$ also contains $c, \gamma$. By the same argument as above, if $G$ is decomposable, the neighborhood of $o$ is as in Figure 48. In this case, 


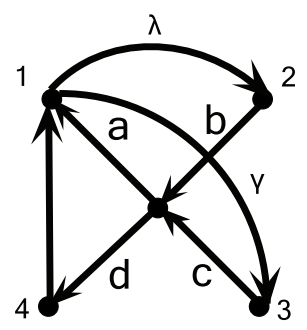

Figure 48

it is a disjoint connected component, and can be obtained by gluing two spikes $\overline{14}, d$ to a diamond that contains $a, b, c, \lambda, \gamma$.

3. Suppose $a$ comes from a diamond. According to the assumption, $a, b, c, \lambda, \gamma$ do not come from the same diamond. Therefore, the diamond containing a must contain $b, d$ with $b$ as its mid-edge. Then node $\mathbf{1}$ is black and $\gamma$ can not be attached, so we get a contradiction.

4. If $a$ comes from a square, it must contain $b, c, d, \lambda, \gamma$. Moreover, nodes $\mathbf{2 , 4}$ and $\mathbf{3 , 4}$ must be connected, which again contradicts our assumption.

To conclude, under the given assumption, $a, b, c, \lambda, \gamma$ come from the same diamond in one of the decomposition of $G$.

Remark 10. If nodes $\mathbf{2 , 3}$ and nodes $\mathbf{3 , 4}$ are connected, and the degrees of nodes $\mathbf{2 , 3}$ are both two, node 4 must coincide with node $\mathbf{1}$. In this case, the neighborhood is shown in Figure 46.

Remark 11. In the above situation, if $G$ is decomposable, we may have more than one decomposition. However, according to the proof of the lemma, there are more than one decomposition only when the neighborhood is a disjoint connected component. We list all such disjoint connected components in Figure 78. If the whole graph coincides with such a disjoint component from this list we know already all the possible decompositions and we do not need to do simplifying replacements. On the other hand, if a decomposable graph does not coincide with any of the graphs in Figure 78, then the decomposition is unique.

Next, we examine the connectivity between node $\mathbf{4}$ and nodes $\mathbf{2 , 3}$.

Assume node $\mathbf{4}$ is connected to both nodes $\mathbf{3}$ and $\mathbf{2}$, see Figure 49. If nodes $\mathbf{3 , 4}$ are connected by an edge directed from node $\mathbf{3}$ to node $\mathbf{4}$, relabel the indices of the nodes in the following way: $\mathbf{4} \rightarrow \mathbf{1}, \mathbf{3} \rightarrow \mathbf{2}, \mathbf{2} \rightarrow \mathbf{3}$ and $\mathbf{1} \rightarrow \mathbf{4}$. Then apply the previous argument. It is similar if nodes $\mathbf{2 , 4}$ are connected by an edge directed from node $\mathbf{2}$ to nodes $\mathbf{4}$. Hence, without loss of generality, assume that edge $\overline{\mathbf{2 4}}$ is directed from node $\mathbf{4}$ to $\mathbf{2}$ and edge $\overline{\mathbf{3 4}}$ is directed from node 4 to $\mathbf{3}$. If none of the nodes in $G$ (except for node $o$ ) is connected to any of the nodes $\mathbf{1}, \mathbf{2}, \mathbf{3 , 4}$, then it is a disjoint connected component.

Suppose we can find a node $x$ which does not coincide with $o$ that is connected to some of the nodes $\mathbf{1 , 2 , 3 , 4}$. We check if there is any node among $\mathbf{1 , 2 , 3 , 4}$ that is connected 


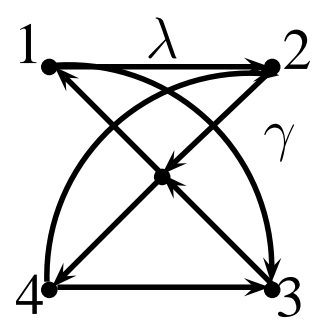

Figure 49

to $x$. Assume $x$ is connected to only one of $\mathbf{1 , 2 , 3 , 4}$. Without loss of generality, assume $x$ is connected to $\mathbf{1}$ by an edge denoted by $\tau$. (Notice that $x$ may be connected to nodes

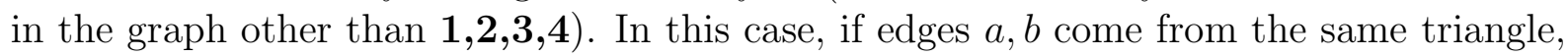
then edges $c, d$ come from another triangle, denoted by $\triangle_{1}$. Moreover, $\tau, \gamma$ come from the same block, which must be a triangle $\triangle_{2}$. Because $x$ is only connected to one of the nodes $\mathbf{1 , 2 , 3 , 4}$, the third edge of $\triangle_{2}$ is annihilated by another edge, denoted by $\eta$. However, node 3 is already black after gluing $\triangle_{2}$ to $\triangle_{1}$, a contradiction. Hence, edges $a, c$ come from the same triangle and $b, d$ come from the same triangle. Therefore, edges $\tau$ and $\lambda$ come from the same block, which is impossible.

Assume that there is a node $x$ which does not coincide $o$ that is connected to only two nodes of the nodes $\mathbf{1 , 2 , 3 , 4}$. Notice that $x$ may be connected to nodes other than $\mathbf{1 , 2 , 3 , 4}$. Up to a relabeling of indices, there are two possible situations: Either $x$ is connected to nodes $\mathbf{1 , 4}$ or to nodes $\mathbf{1 , 3}$.

First, suppose $x$ is connected to nodes $\mathbf{1 , 4}$ by edges $\tau, \eta$ respectively. If $a$ comes from a spike, then $\tau, \lambda, \gamma$ come from the same block which is a diamond. Judging by the directions of $\lambda, \gamma, \tau$ must be the mid-edge. Therefore, node $x$ must coincide with node $o$. This contradicts our assumption.

Suppose $a$ comes from a diamond. Since the degrees of node $\mathbf{1}$ and $o$ are at least $4, a$ must be the mid-edge. Therefore, the diamond must contain $\lambda, \gamma, b, d$. Moreover, the degrees of node 2,3 must be two, a contradiction.

We can also rule out the possibility that $a$ comes from a square since both its endpoints have degree 4 . Thus, a must come from a triangle. Otherwise, $G$ is indecomposable. Suppose $a, \tau$ come from the same triangle, then the third edge of this block is annihilated by another edge, denoted by $\delta$. Hence $\delta, \eta, b, c, d$ come from the same block, which is impossible. If $a, b$ come from the same triangle, then $c, d$ come from another triangle, denoted by $\triangle_{1}$. Thus, $\tau, \gamma$ come from the same block, which must be a triangle, denoted by $\triangle_{2}$. Notice that its third edge is annihilated since $x$ is connected to only two of the nodes $\mathbf{1}, \mathbf{2 , 3 , 4}$. But this is impossible since node $\mathbf{3}$ is already black after gluing $\triangle_{1}$ to $\triangle_{2}$. The similar argument shows that $a, c$ can not come from the same triangle. Thus, the graph is indecomposable when $x$ is connected only to nodes $\mathbf{1 , 4}$.

Assume that $x$ is connected to nodes $\mathbf{1 , 3}$ by edges $\tau, \eta$ respectively. If $a, c$ come from the same triangle, then $b, d$ come from another triangle, denoted by $\triangle_{1}$. Thus, $\tau, \lambda$ come from another block. This block can not be a diamond since $\lambda$ must then be the mid-edge 


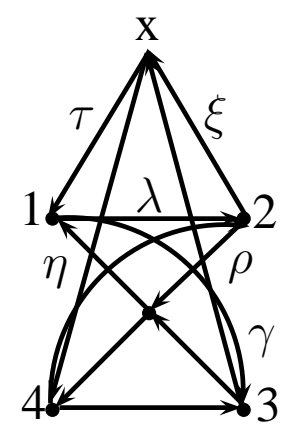

Figure 50

and the boundary edge $\overline{x \mathbf{2}}$ is annihilated, which is impossible. Hence this block must be a triangle, denoted by $\triangle_{2}$. Since $x$ is connected only to two of nodes $1,2,3,4$, the third edge of $\triangle_{2}$ is annihilated. However, node 2 is already black after gluing $\triangle_{1}$ to $\triangle_{2}$. This is a contradiction. Therefore if $G$ is decomposable, $a, b$ must come from the same triangle and $c, d$ from another triangle. Apply the corresponding replacement as in Figure 36.

Assume $x$ is connected to at least three of the nodes $\mathbf{1 , 2 , 3 , 4}$. Up to an index relabeling, there are two cases: either $x$ is connected to nodes $\mathbf{1 , 2 , 3}$ or $x$ is connected to nodes $\mathbf{1 , 3 , 4}$. Suppose $x$ is connected to nodes $\mathbf{1 , 3 , 4}$ by $\tau, \rho, \eta$ respectively. Assume that $a, b$ come from the same triangle, then $c, d$ come from one triangle too. Thus, $\eta, \overline{\mathbf{2 4}}$ come from the same block, which must be a triangle, and so node $x$ and node $\mathbf{2}$ must be connected according to previous argument. The argument is similar when $a$ and $c$ come from the same triangle. In both cases, node $o$ is contained in a neighborhood in $\mathcal{S}_{o}$ which is a disjoint connected component, see Figure 50. Otherwise, $G$ is indecomposable.

Assume $x$ is connected to nodes $\mathbf{1 , 2 , 3}$ by $\tau, \xi, \rho$ respectively. Assume that $a, b$ come from the same triangle, then $c, d$ come from another triangle, denoted by $\triangle_{1}$. Therefore, $\tau, \gamma, \rho$ must come from the same triangular block, denoted by $\triangle_{2}$. Notice that node $x$ is black after gluing $\triangle_{1}$ to $\triangle_{2}$, node $x$ and node 4 must be connected by the third edge of $\triangle_{2}$. Similarly, assume that $a, c$ come from the same triangle, we will get the same neighborhood (see Figure 50). In this case, the neighborhood is a disjoint connected component, Otherwise, the graph is indecomposable.

Assume next that node $\mathbf{4}$ is connected only to one of nodes $\mathbf{2 , 3}$.

Suppose node $\mathbf{4}$ is connected to node $\mathbf{3}$. In this case, if edge $\overline{\mathbf{3 4}}$ is directed towards node $\mathbf{4}$, then edges $c, d, \overrightarrow{\mathbf{3 4}}$ can not form a triangular block. This means edges $b, d$ and edges $a, c$ must come from two triangles, otherwise the graph is indecomposable. By assumption, nodes $\mathbf{2 , 4}$ are not connected, hence the edge with nodes $\mathbf{2 , 4}$ as its endpoints must be annihilated by another edge, denoted by $\eta$. Hence, $\eta, \lambda$ come from the same block which must be a triangle. So node 4 is black before attaching edge $\overrightarrow{\mathbf{3 4}}$. This is a contradiction. In this case, the graph is indecomposable.

If edge $\overline{34}$ is directed towards node $\mathbf{3}$, there are two possibilities: edges $c, d, \overrightarrow{\mathbf{4 3}}$ form a triangular block or edges $b, d$ come from a triangle $\triangle$.

Suppose it is the latter case, the edge of $\triangle$ that connects nodes $\mathbf{2 , 4}$ is annihilated by another edge $\tau$. This forces edges $\tau, \overrightarrow{\mathbf{4 3}}, \lambda$ to form a block, which is impossible. So edges 


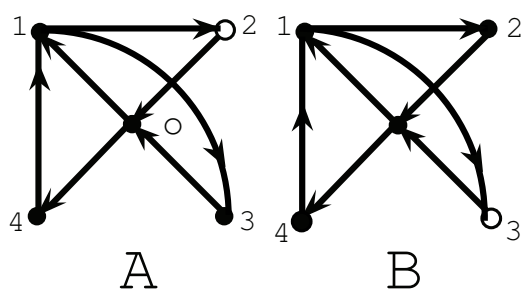

Figure 51

$c, d, \overline{34}$ form a triangle. Otherwise, the graph is indecomposable. Hence, edges $c, d, \overrightarrow{43}$ form a triangular block. We apply the same replacement as in Figure 36.

Finally, assume that nodes $\mathbf{3 , 4}$ and nodes $\mathbf{2 , 4}$ are disconnected. Then one edge of the triangular block that contains edge $d$ is annihilated by another edge, denoted by $\tau$. If $a, b$ come from the same triangle, then $\tau$ connects nodes $\mathbf{3 , 4}$. Therefore, $\gamma, \tau$ must form a triangle, which means that nodes $\mathbf{1 , 4}$ must be connected and nodes $\mathbf{2 , 3}$ are disconnected. Similarly, if $b, d$ come from a triangle, nodes $\mathbf{2 , 4}$ must be connected by $\tau$, Thus, nodes $\mathbf{2 , 3}$ must be disconnected and nodes $\mathbf{1 , 4}$ are connected. (Figure 51). Notice that in picture A, node $\mathbf{2}$ may have degree larger than two, and in picture B, node $\mathbf{3}$ may have degree larger than 2. Thus, it suffices to examine the degrees of nodes $\mathbf{2 , 3}$ to determine whether edges $a, b$ or edges $a, c$ come from a triangular block, and then apply the corresponding replacement. To be more precise, if degree of node $\mathbf{2}$ is at least 3, node $o$ is contained in a neighborhood in $\mathcal{S}_{o}$ as shown in picture A; if the degree of node $\mathbf{3}$ is at least 3 , node $o$ is contained in a neighborhood in $\mathcal{S}_{o}$ as shown in picture $\mathrm{B}$; if both nodes $\mathbf{2 , 3}$ have degree 2 , either decomposition is possible, and the neighborhood is a disjoint connected component.

\subsection{Node 1 is Connected to Node 2 but Disconnected from Node 3}

Assume that nodes $\mathbf{1 , 2}$ are connected by an edge $\lambda$, but nodes $\mathbf{1 , 3}$ are not connected. If $\lambda$ is directed from node $\mathbf{2}$ to node $\mathbf{1}$, then $a, b, \lambda$ do not form a triangular block. Moreover, $a$ does not come from a diamond. If $a$ comes from a spike, $b, c, d$ must come from a diamond and node $\mathbf{4}$ is connected only to nodes $\mathbf{2 , 3}$. By assumption, nodes $\mathbf{1 , 2}$ are connected, node 1 must coincide with node $\mathbf{4}$. This means nodes $\mathbf{1 , 3}$ are connected, which contradicts our assumption. Therefore, a must come from a triangle that does not contain $b, \lambda$, hence the block must contain $c$. The third edge in that triangle is annihilated by another edge, denoted by $\tau$. Moreover, $\tau$ is directed from node $\mathbf{3}$ to node $\mathbf{1}$. Thus, $\tau, \lambda$ come from the same block. However, there is no such block with such directions, so in this case the graph is indecomposable.

Assume next that $\lambda$ is directed from node $\mathbf{1}$ to node $\mathbf{2}$.

If $a$ comes from a spike, $b, c, d$ come from the same block, which must be a diamond. Since nodes $\mathbf{1 , 2}$ are connected, node 1 must coincide with nodes $\mathbf{4}$. Therefore nodes $\mathbf{1 , 3}$ are connected, a contradiction. 
Suppose $a$ comes from a diamond. Note that the degree of node $o$ is four, it must be one endpoint of the mid-edge. Taking into consideration the directions of $a, b, c, d$, there are three cases.

- $a$ is the mid-edge of the diamond. In this case, node $\mathbf{2}$ must be contained in the same diamond. Since node $\mathbf{2}$ is not an endpoint of the mid-edge, it must have degree two, a contradiction.

- $b$ is the mid-edge of the diamond. In this case, $d$ must be contained in the diamond. Therefore, nodes 3,4 are disconnected. Moreover, the degrees of nodes $\mathbf{1}$ and $\mathbf{4}$ are both two.

- $c$ is the mid-edge of the diamond. In this case $d$ must be contained in the diamond, and nodes 1,3 must be connected, a contradiction.

Lemma 7. Assume that nodes $\mathbf{1 , 2}$ are connected by edge $\lambda$ directed from node $\mathbf{1}$ to node 2, nodes 1,3 are disconnected, nodes 2,4 are connected by an edge directed from node 4 to 2 , and that the degrees of nodes $\mathbf{1 , 4}$ are two.

(1) If $G$ is decomposable and nodes 2,3 are disconnected, then a comes from a diamond containing $a, b, d, \lambda$ and $c$ comes from a spike.

(2) Assume nodes 2,3 are connected by an edge directed from node 2 to 3 :

(a) If the degree of node 3 is two, then o is contained in a neighborhood in $\mathcal{S}_{o}$, and this neighborhood is a disjoint connected component.

(b) If the degree of node 3 is at least three, then the graph is not decomposable.

Proof. (1): Suppose nodes 2,3 are disconnected and the statement is false. It is easy to rule out the possibility that $a$ comes from a square or a fork.

If $a$ comes from a spike, $b, c, d$ comes from a diamond and node $\mathbf{2}$ is black. Thus, $\lambda$ can not be attached unless node $\mathbf{1}$ coincides with node $\mathbf{4}$. Thus the degree of node $\mathbf{1}$ is four. This contradicts the assumption that degree of node $\mathbf{1}$ is 2 . Suppose edge a comes from a diamond. Since the statement is false, the diamond must contain $a, b, c$. Hence node $\mathbf{1}$ must be connected to node $\mathbf{3}$, which is a contradiction to our assumption.

If $a, b$ come from a triangle, $c, d$ also come from a triangle denoted by $\triangle$. Thus, the third edge of $\triangle$ is annihilated by another edge, denoted by $\eta$. Hence $\eta, \overline{\mathbf{2 4}}$ come from the same block, which must be a triangle. Thus, node 2,3 must be connected, a contradiction. If $a, c$ come from a triangle, then the third edge of this triangle is annihilated by another edge, again denoted by $\eta$. Hence $\eta, \lambda$ must come from the same block, which must be a triangle. Hence nodes $\mathbf{2 , 3}$ must be connected, a contradiction.

(2): Assume nodes $\mathbf{2 , 3}$ are connected by an edge directed from node $\mathbf{2}$ to $\mathbf{3}$. It suffices to assume that $a$ comes from a triangle or a diamond. Suppose $a$ comes from a triangle, by a previous argument, all nodes in this neighborhood must be black, which proves $(a)$. If $a$ comes from a diamond, the degree of node $\mathbf{3}$ is three. The only possibility to obtain a decomposition is to glue a triangle or diamond on nodes $\mathbf{2 , 3}$. In either case, the degree 


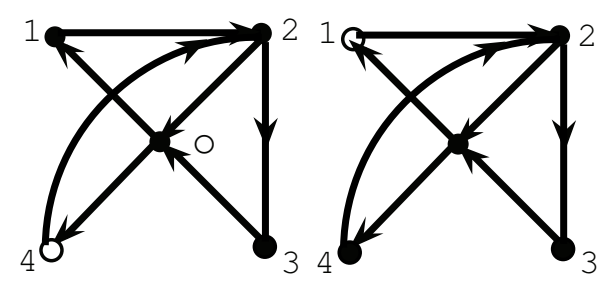

Figure 52

of node $\mathbf{2}$ is larger than 4 . This contradicts our assumption in the section that the degree of any node of $G$ is at most 4 . Hence the graph is indecomposable, and it proves $(b)$.

Suppose node 1 or node 4 has degree at least three, then $a$ does not come from a diamond. Moreover, we can rule out the possibility that $a$ comes from a square, since this will force node 1 to be connected to node 3 with an edge $\overrightarrow{\mathbf{1 3}}$. Hence $a$ must come from a triangle. There are two cases: (1). edges $a, b$ are in the same triangle; (2). edges $a, c$ are in the same triangle. In the first case, edges $c, d$ are in the same triangle. In this case, apply the same replacement as in Figure 36. In the second case, edges $b, d$ are in the same triangle. Denoted the triangle containing edges $a, b$ by $\triangle$. The edge in $\triangle$ that connects nodes $\mathbf{1 , 3}$ is annihilated by another edge, denoted by $\lambda$. Thus, $\gamma, \lambda$ come from the same block, and it must be a triangle. This means that nodes $\mathbf{2 , 3}$ are connected. Moreover, in this case, nodes 2,4 must be connected by an edge directed from node 4 to 2 and nodes $\mathbf{1 , 2 , 3}$ are black. Notice that nothing has been glued to node $\mathbf{4}$ yet, this fact is used to distinguish the second case.

Lemma 8. Suppose that graph $G$ is decomposable and node $\mathbf{1}$ is connected to node 2 by an oriented edge $\overrightarrow{12}$. Assume further that nodes 1,3 are disconnected:

(a) Suppose nodes 2,4 and nodes 2,3 are connected. If the degree of node 4 is at least 3 , then a, come from one triangle and $b, d$ come from another triangle. If degree of node 1 is at least 3 , then $a, b$ come from the same triangle and $c, d$ come from the same triangle (Figure 52). If the degrees of both nodes 1 and 4 are 2, then o is contained in a neighborhood in $\mathcal{S}_{o}$, and this neighborhood is a disjoint connected component.

(b) If nodes 2,4 or 2,3 are disconnected, then a, $b$ come from one triangle, and $c, d$ come from another triangle.

Proof. According to the previous argument, it suffices to prove (a). Suppose nodes 2,4 and nodes $\mathbf{2 , 3}$ are connected. If $a, b$ come from one triangle, then $c, d$ come from another triangle. Moreover, edges $\overline{\mathbf{2 4}}, \overline{\mathbf{2 3}}$ must come from the same block, which must be a triangle. The third edge of this triangle annihilates $\overline{\mathbf{3 4}}$. Therefore, node $\mathbf{4}$ have degree 2 . so, if the degree of node 4 is at least 3, a,c must come from one triangle. Otherwise, the graph is indecomposable. The rest of part $(a)$ follows from the previous argument. 


\subsection{Node 1 is Disconnected from Nodes 2,3}

Assume that neither nodes $\mathbf{1 , 2}$ nor nodes $\mathbf{1 , 3}$ are connected. Without loss of generality, we can assume that neither nodes $\mathbf{3 , 4}$ nor nodes $\mathbf{2 , 4}$ are connected. Otherwise, we can relabel the indices of boundary nodes and apply the previous argument.

Assume that $a, b$ come from the same triangle. Then the third edge of it is annihilated by another edge, denoted by $\lambda$. This edge $\lambda$ can be a part of a spike, a triangle, or the mid-edge of a diamond block. If it comes from a triangle or a diamond, nodes 1,2 must both be connected to another node $x$. Conversely, it is possible to determine whether $a, b$ or $a, c$ come from the same triangle by considering nodes connecting some of the nodes $1,2,3,4$.

Suppose none of the nodes $1,2,3,4$ is connected to any other node except for $o$, then the neighborhood is a disjoint connected component.

Assume that nodes $\mathbf{1 , 4}$ or $\mathbf{2 , 3}$ are both connected to the same node $x$. We also assume that vertex $x$ is distinct from nodes $\mathbf{1 , 2 , 3 , 4}$, and $o$. Without loss of generality, assume nodes 1,4 are connected to $x$. Let $\alpha:=\overline{\mathbf{1} x}$ and $\beta:=\overline{\mathbf{4} x}$. If $a, d$ come from the same block, it must be a diamond. Hence $b, c$ come from another diamond. Since the degree of $o$ is 4 , the mid-edges of these two diamonds annihilate each other. Then the neighborhood is a disjoint connected component, see Figure 37. If we rule out this case, $a, d$ must come from two blocks. By the previous argument, neither $a$ nor $d$ comes from the a diamond. Thus, they must come from triangular blocks. Moreover, the triangle that contains a must contain $c$ or $b$. Without loss of generality, assume $c$ is contained in such triangle $\triangle$. Then the third side of $\triangle$ is annihilated by another edge, denoted by $\tau$. Similarly, $b, d$ come from another triangle $\triangle_{1}$. The third edge of $\triangle_{1}$ is annihilated by an edge denoted by $\eta$. Then $\alpha, \tau$ come from the same block, which must be a triangle. If node $\mathbf{3}$ and $x$ are not connected, the third edge of the triangle containing $\alpha, \tau$ must be annihilated. This is impossible since node $\mathbf{3}$ is already black. Therefore, in this case, the graph is indecomposable. If nodes $\mathbf{3}$ and $x$ are connected by an edge $\gamma$, then $\alpha, \tau, \gamma$ form a triangle if their directions match a triangle, otherwise, the graph is indecomposable. Notice that $\beta, \eta$ must come from the same block. Thus, it must be a triangle if their directions match, otherwise, the graph is indecomposable. In this case, nodes $x, \mathbf{2}$ must be connected, and the neighborhood is a disjoint connected component, see Figure 53. In this case, there is an alternative decomposition, see Figure 54.

Assume next that $x$ is connected to nodes $\{\mathbf{1}, \mathbf{2}\}$ (resp. $\{\mathbf{1}, \mathbf{3}\},\{\mathbf{3}, \mathbf{4}\},\{\mathbf{2}, \mathbf{4}\}$ ), we claim that $a, b$ (resp. $a, c, c, d, b, d$ ) come from one triangle, therefore $c, d$ (resp. $b, d, a, b, a, c)$ must come from another triangle.

Assume that nodes $\mathbf{1 , 2}$ are connected to node $x$ and that $a, b$ do not come from the same triangle in any decomposition of $G$. Denote $\alpha:=\overline{\mathbf{1} x}, \beta=\overline{\mathbf{2 x}}$. Notice that from the previous argument, $a$ does not come from a spike, a fork, a diamond or a square. So it must be contained in a triangular block $\triangle$. If $\triangle$ contains $\alpha$, then the third edge is annihilated by another edge, denoted by $\tau$. Hence, $\tau, b, c, d$ come from the same block which must be a square, also nodes $o, x$ must be colored white in that block. This is impossible, so $\triangle$ does not contain $\alpha$, and therefore must contain $c$. Then the third edge 


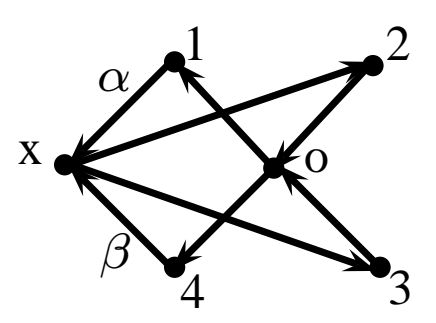

Figure 53

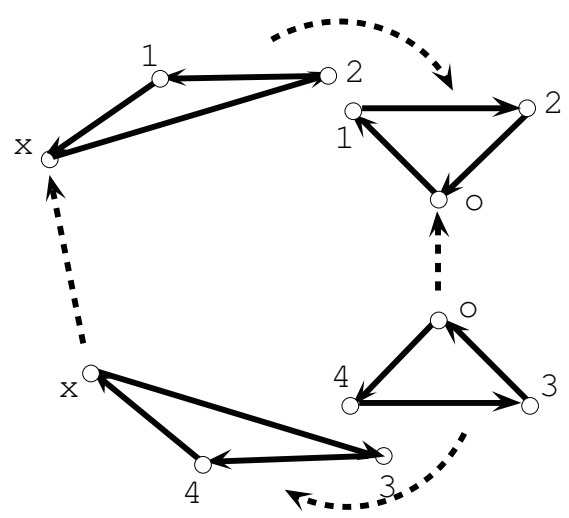

Figure 54

must also be annihilated by another edge, again denoted by $\tau$. In this case, $\tau, \alpha$ must come from the same block, which must be a triangle $\triangle_{1}$. If nodes 3 and $x$ are connected, we will get a neighborhood similar to the one in Figure 53. As we already know, there is an alternative decomposition in which $a, b$ come from the same triangle. If nodes $\mathbf{3}$ and $x$ are not connected, then the third edge of $\triangle_{1}$ is annihilated by another edge. However, node 3 is already black after gluing $\triangle_{1}$. This means the third edge of $\triangle_{1}$ can not be annihilated. This is a contradiction. Therefore, $a, b$ come from the same triangle in a decomposition of $G$. Otherwise, $G$ is not decomposable.

Remark 12. If $x$ is connected to nodes $\mathbf{1 , 2}$ and the graph is decomposable, either $G$ has a unique decomposition in which $a, b$ comes from a triangular block and $c, d$ comes from another, or $G$ is the graph as in Figure 53.

\section{Simplification on Nodes of Degree Three}

Assume the neighborhoods of nodes of degree at least 4 are all simplified, and every node in the graph has degree at most 3 . We focus on the nodes of degree 3.

\subsection{All edges have the same direction.}

Without loss of generality, assume that the edges are all directed outwards, (see Figure 55) Suppose one of them (denoted by a) comes from a triangle. Since $\operatorname{deg}(o)=3$, and neither of the remaining two edges comes from the same triangle, the incoming edge incident to $o$ in the same triangle must be annihilated. Denote this edge as $e$. Then $e$ must be annihilated by an outward edge from another block. This block must contain the remaining outward edges $b$ and $c$. But no such block exists, a contradiction. Hence edge $a$ is not contained in a triangular block. By symmetry, none of the three edges comes from a triangle.

If one of them comes from a fork, one of the remaining two edges must also belong to the same fork. Thus, the third edge must come from a single spike. (Figure 56) Otherwise, 
the graph is indecomposable. Replace the neighborhood with Figure 57. By Lemma 1, this replacement is consistent.

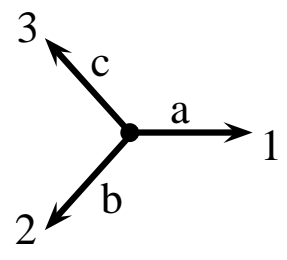

Figure 55

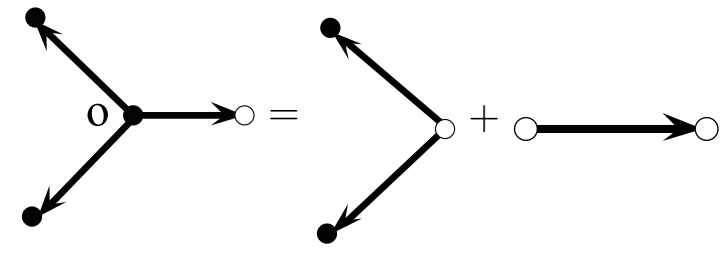

Figure 56

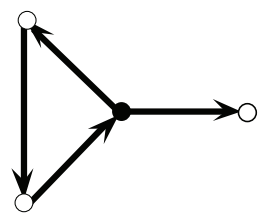

Figure 57

Remark 13. In order to apply the replacement, we need to determine which two edges come from a fork. This can be done by checking the degrees of boundary nodes. If one of the boundary nodes has degree more than 1 , the corresponding edge must come from a spike and the remaining two edges form a fork. If all boundary nodes have degree 1, we have a disjoint connected component and the decomposition is non-unique. If at least two of the boundary nodes have degrees more than 1, the graph is indecomposable.

If one of the edges, denoted by $a$, comes from a diamond, denoted by $\diamond$, then one of the remaining nodes, denoted by $b$, must come from the same diamond. Thus the third edge $c$ is not contained in the same block. Since the degree of $o$ is 3 , the mid-edge in $\diamond$ must be annihilated by another edge, $e$ say, directed away from $o$. Thus, $c, e$ come from the same block. Since the degree of $o$ is 3 , the block containing $c, e$ must be a triangle. However, the directions of these two edges do not match a triangle. This is a contradiction.

To conclude, if all three edges incident to a node are all directed inwards or outwards and the graph is decomposable, the neighborhood must be obtained from gluing a fork and a spike.

\subsection{Two outward edges and one inward edge}

Assume edge $a$ is directed inwards with nodes $\mathbf{1}$ and $o$ as endpoints, see Figure 58. If $a$ comes from a spike, the remaining two edges must come from a fork. If a comes from a diamond $\diamond$, the block must contain at least $b$ or $c$ since only the mid-edge can be annihilated in a diamond. Assume $b$ is contained in this block. The directions of $a, b$ force $c$ to be contained in $\diamond$, as shown in Figure 59. Since all nodes in $G$ has degree at most 3, this diamond must be a disjoint connected component. Otherwise, $G$ is indecomposable.

Assume $a$ comes from a triangle $\triangle$, there are two cases:

Case 1: $\triangle$ contains neither of $b, c$. Then $b, c$ must come from a diamond or a fork. In the latter, the remaining edge of $\triangle$ that is incident to $o$ must be annihilated. This forces $o$ to be a black node even before $b, c$ are attached. This is a contradiction, so $b, c$ come from a diamond $\diamond$. Notice that the mid-edge in $\diamond$ should be annihilated by an edge of $\triangle$, as shown in Figure 60. Simplify the neighborhood by removing the diamond block 


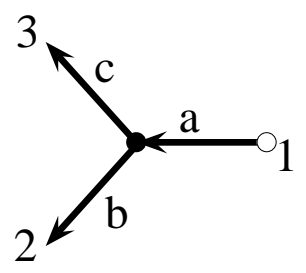

Figure 58

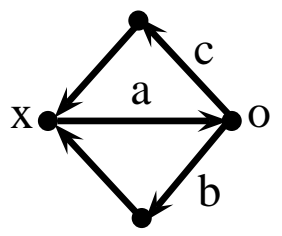

Figure 59

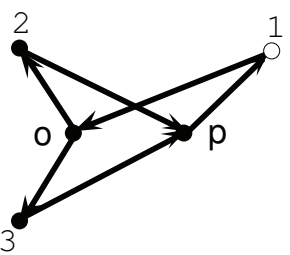

Figure 60

and leaving the triangle $\triangle$ containing $a$ (see Figure 61).

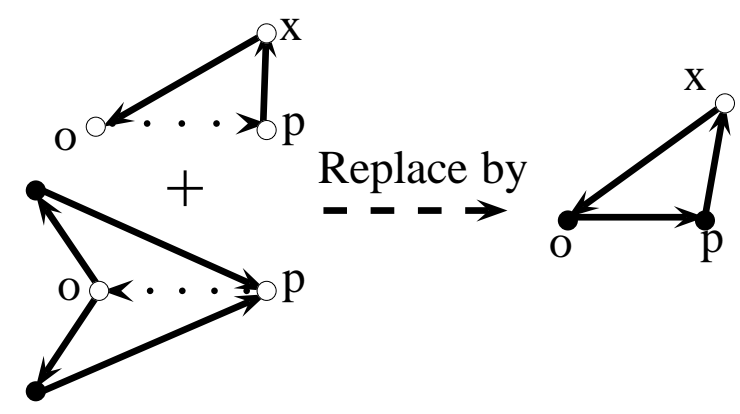

Figure 61

Case 2: $\triangle$ contains one of $b, c$. Without loss of generality, assume it is $b$. Then $c$ must come from a spike.

Let us take a deeper look at Case 2. Assume that the third edge of $\triangle$ is $d$. Then there are two possibilities.

(a.) Edge $d$ is annihilated in the graph.

(b.) Edge $d$ is not annihilated in the graph (Figure 62).

Next, start with case $\mathbf{a}$. There are three ways to annihilate $d$.

Case (a1): Edge $d$ is annihilated by a single spike, see Figure 63. Then replace this neighborhood by the one in Figure 64. By Lemma 1, this replacement is consistent.

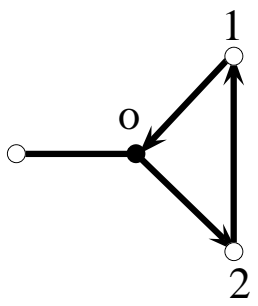

Figure 62

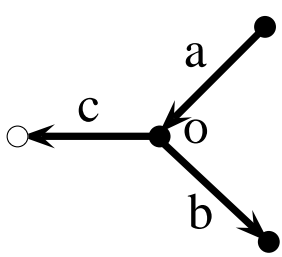

Figure 63

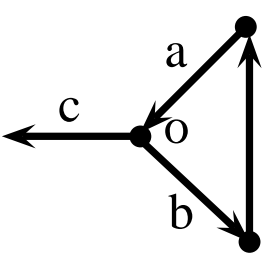

Figure 64 
Case (a2): Edge $d$ is annihilated by one edge of a triangle, see Figure 65. If $p$ is connected to $o$ via edge $c$, then this graph forms a disjoint connected component (Figure 66). Otherwise, $G$ is indecomposable. If $c$ does not connect $o$ with $p$, and there is nothing else connected to $p(\operatorname{deg}(p)=2)$. Then we replace the neighborhood in Figure 65 with the one in Figure 64. If $c$ does not connect $p$, and $\operatorname{deg}(p)=3$, as shown in Figure 67, there are two cases:

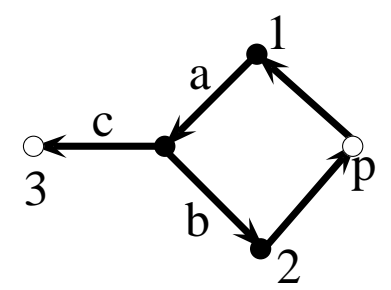

Figure 65

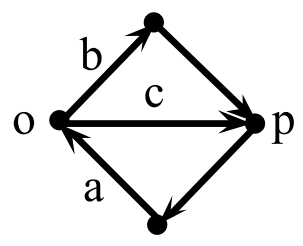

Figure 66

- In Figure 67, suppose node 3 coincides with node $x$, edge $\overline{p x}$ is directed from $x$ to $p$ and $\operatorname{deg}(1)=2$, the neighborhood in Figure 67 coincides with Figure 60. In this case, if graph $G$ is decomposable, the neighborhood is a disjoint connected component.

- Suppose node 3 does not coincide with node $x$. Then the neighborhood can replaced by Figure 68. It is similar if edge $\overline{p x}$ is directed from $p$ to $x$. This replacement is consistent by the previous lemma.

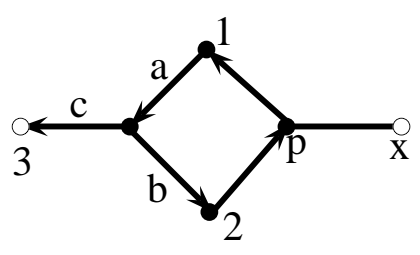

Figure 67

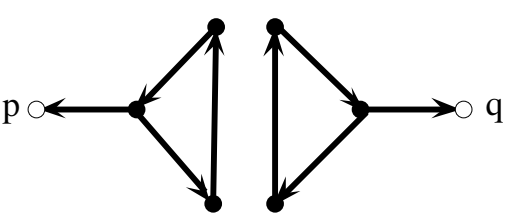

Figure 68

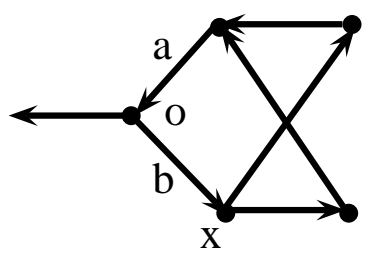

Figure 69

Case (a3): Assume $d$ is annihilated by the mid-edge of a diamond, see Figure 69 . Replace the neighborhood by the one in Figure 64 as well.

Next, let us discuss case $\mathbf{b}$. If $d$ is not annihilated, there are three subcases: 
(b1): Both nodes $\mathbf{1 , 2}$ have degree two. By Lemma 1, node $o$ must be contained in a neighborhood in $\mathcal{S}_{o}$ that come from a spike and a triangle.

(b2): One of the nodes $\mathbf{1 , 2}$ has degree two and the other one has degree three. Assume the degree of node $\mathbf{2}$ is two, and the degree of node $\mathbf{1}$ is three. In this case, $o$ is contained in a neighborhood as shown in Figure 70.
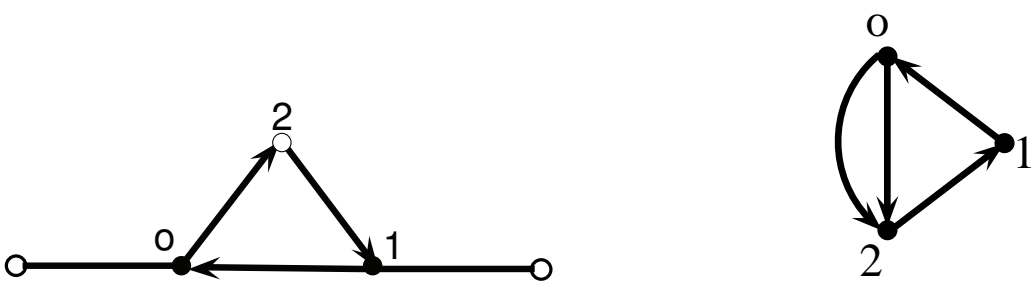

Figure 70

(b3): Both nodes $\mathbf{1 , 2}$ have degree three. In this case, we count the number of nodes that are connected to nodes $o$ and $\mathbf{1 , 2}$, denoted by $n$.

- Suppose $n=3$, the only possible decomposable situation is given in Figure 71 .

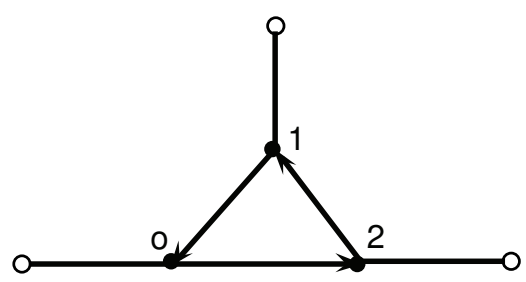

Figure 71

- Suppose $n=2$. One of the exterior nodes is connected to two of the nodes $o, \mathbf{1 , 2}$. Denote this node by $x$. If $x$ is connected to nodes $\mathbf{1 , 2}$ (resp. $o, \mathbf{1}$ or $o$, $\mathbf{2}$ ), then the other exterior node is connected to nodes $o$ (resp. node $\mathbf{2}$ or node 1). In this case, edges $\overline{x \mathbf{1}}, \overline{x \mathbf{2}}$ (resp. $\overline{x o}, \overline{x \mathbf{1}}$ or $\overline{x o}, \overline{x \mathbf{2}}$ ) come from two spikes and the degree of $x$ must be two (see Figure 72).

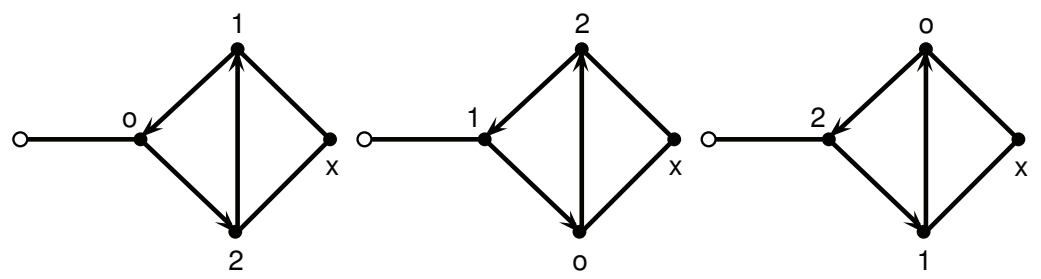

Figure 72 
- Suppose $n=1$. Notice that we assume that the degree of nodes $0, \mathbf{1 , 2}$ are all three. So there are two cases, as shown in Figure 73. Note that Figure 73A is indecomposable, so the only decomposable neighborhood is Figure 73B.

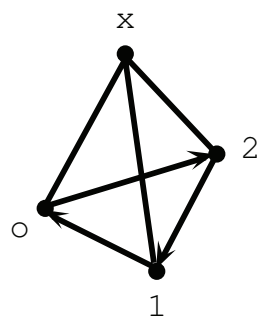

A

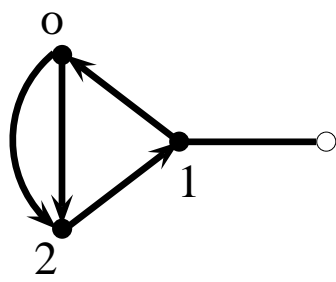

B

Figure 73

To sum up:

1. Every node in $G$ has degree at most 3 .

2. Consider all nodes of degree 3. If all of them fall into the decomposable categories (Figure 74), then either the neighborhood forms a disjoint connected component that can be easily decomposed, or we can apply the corresponding replacement. If graph $G$ contains any neighborhood (up to a reversion of edge directions) that is unlisted in Figure 74 , the graph is not decomposable.

Remark 14. We can reverse the directions of all edges to get another 14 neighborhoods in decomposable graph.

Remark 15. Note that the degree of node $o$ is not increased in any replacement.

In some of the cases in Figure 74, the neighborhood of the target node $o$ contains some other nodes of degree 3 . The algorithm covers the analysis of the neighborhood of these nodes in the following manner:

- In picture 2 in Figure 74, node $x$ has degree 3. Node $x$ is contained in a neighborhood in $\mathcal{S}_{x}$ that is isomorphic to the one in picture 2 by a complete reversion of edge directions (node $x$ mapped to node $o$ ). Node $p$ in picture 4 is contained in neighborhood in $\mathcal{S}_{p}$ isomorphic to the neighborhood of $o$ in the same picture by a reversion of edge directions. Similarly, a neighborhood of node $p$ in picture 6 (resp. node $y$ in 9 and 10) is also isomorphic to a neighborhood of node $o$ in picture 6 (resp, node $o$ in picture 9 and 10).

- Node $x$ in picture 5 of Figure 74 is contained in a neighborhood isomorphic to the one in picture 4 . To be more specific, the neighborhoods of $x$ in picture 5 is the neighborhood of $o$ in picture 4 .

- Picture 9 is a part of picture 10. Note the replacement for picture 9 is the same as the replacement for picture 10. Therefore, whether node $o$ in picture 9 or 10 is examined first does not affect the result of the algorithm. 


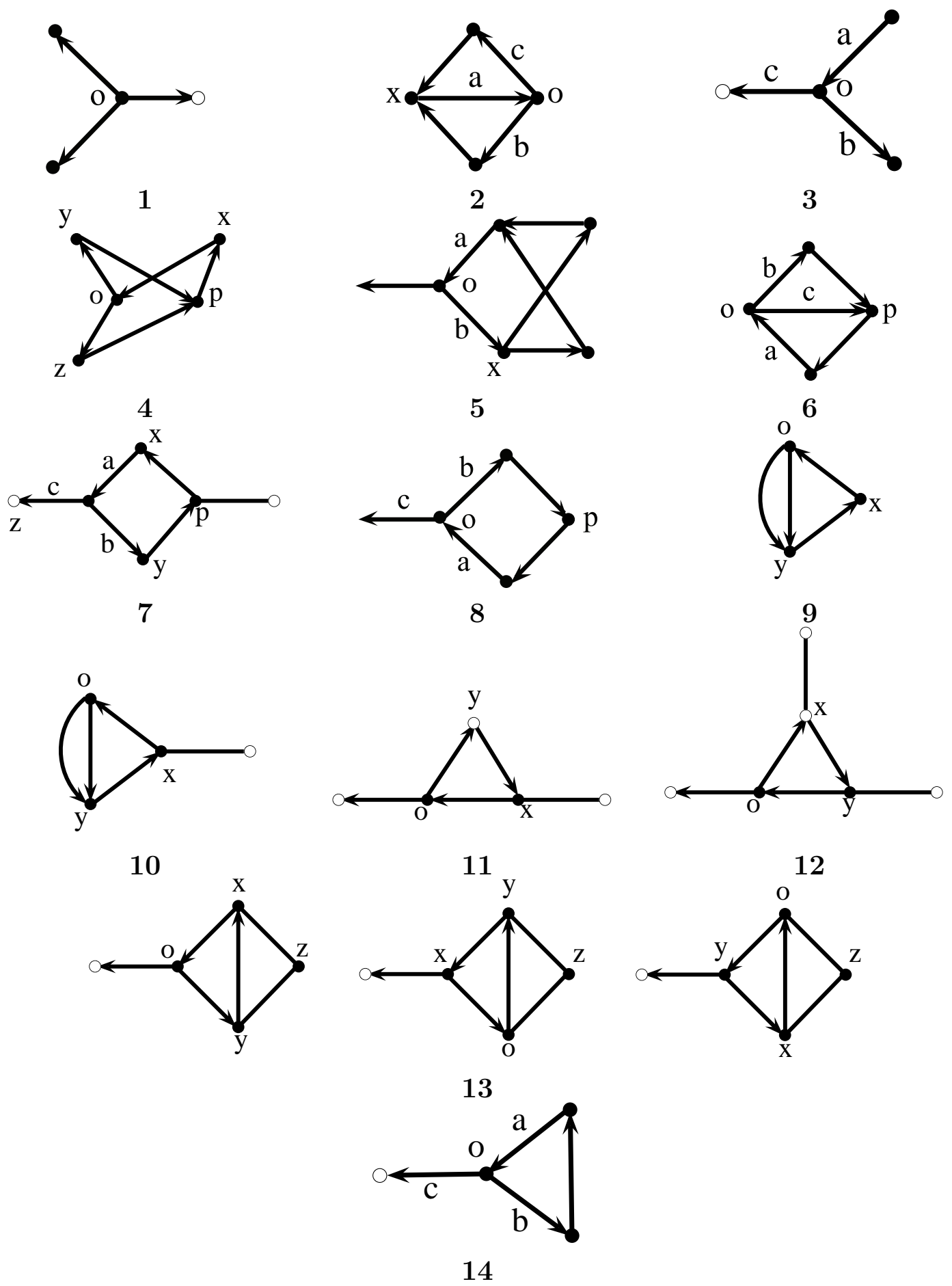

Figure 74: All decomposable cases for degree 3 


\subsection{Determine the Decomposition}

In the previous section, we have found all possible neighborhoods in $\mathcal{S}_{o}$ for node o of degree 3 in a decomposable graph. In this section, we want to determine if a node $o$ is contained in a neighborhood in $\mathcal{S}_{o}$, and which neighborhood it is. This is done by checking two things:

- The number of nodes (other than nodes $\mathbf{1 , 2 , 3}$ and $o$ ) that are connected to some of the nodes $\mathbf{1 , 2 , 3}$. Denote this the number by $n$.

- The direction of edges connecting $o$, its boundary nodes and other nodes that are connected to nodes $\mathbf{1 , 2 , 3}$

If all three edges incident to $o$ have the same direction, the only possible neighborhood in a decomposable graph comes from gluing a fork to a spike. Moreover, $n$ is 0 or 1 . If $n=1$, there is only one node that differs from $o$ and is connected to nodes $\mathbf{1 , 2 , 3}$. Denote it by $x$. If $x$ is connected to node $\mathbf{1}$ (resp. node $\mathbf{2}$ or $\mathbf{3}$ ), then edge $\overline{o \mathbf{1}}$ (resp. $\overline{o \mathbf{2}}$ or $\overline{o \mathbf{3}}$ ) comes from a spike and edges $\overline{o \mathbf{2}}, \overline{o \mathbf{3}}$ (resp. $\overline{o \mathbf{1}}, \overline{o \mathbf{3}}$ or $\overline{o \mathbf{1}}, \overline{o \mathbf{2}}$ ) come from a fork.

We focus on the case when the node $o$ has one incoming and two outgoing edges. Note that the remaining case is when there is one edge going away from node $o$ and two edges going towards node $o$. The latter case can be analyzed by reversing direction of all edges and using the following argument.

Assume node $\mathbf{1}$ is incident to the inward edge. Denote edges $\overline{o \mathbf{1}}, \overline{o \mathbf{2}}, \overline{o \mathbf{3}}$ by $a, b, c$ respectively. By Figure 74, $n \leq 3$.

Suppose $n=0$. By the previous discussion, if the graph is decomposable, we can only have neighborhoods as in Figure 75. After reversing all directions, we can get another four possible cases.
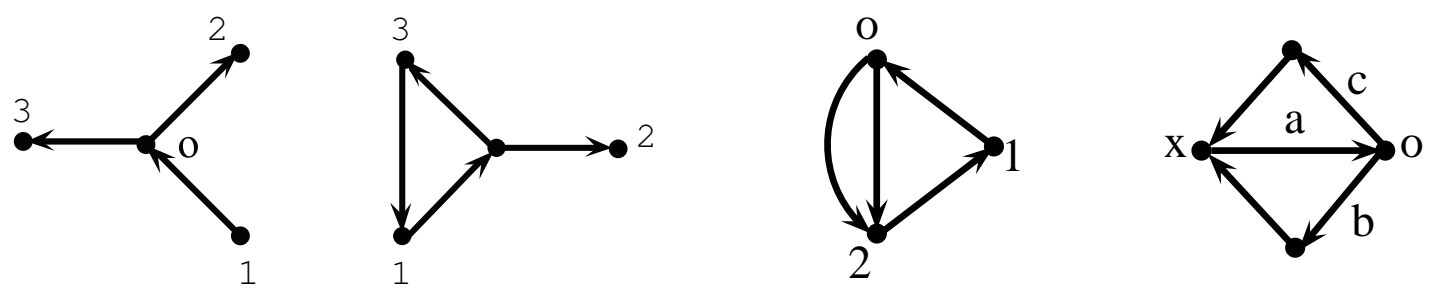

Figure 75

Next, suppose $n=1$. Denote this node by $x$. Assume $x$ is connected to all nodes 1,2,3. There are two cases:

- If $\operatorname{deg}(\mathbf{1})=3$, edges $b, c$ must come from the same diamond and edge $a$ comes from a triangle. See Figure 76 for directions of edges. Note that it is exactly picture 4 in Figure 74.

- If $\operatorname{deg}(\mathbf{1})=2$, the neighborhood is a disjoint connected component, there are two possible decomposition: (1). a comes from a triangular block and $b, c$ come from a diamond; (2). $a, b$ come from one triangular block, and edges $\overline{\mathbf{2} p}$ and $\overline{\mathbf{1} p}$ come from another triangular block, and edges $c$ and $\overline{\mathbf{3} p}$ come from two spikes. 


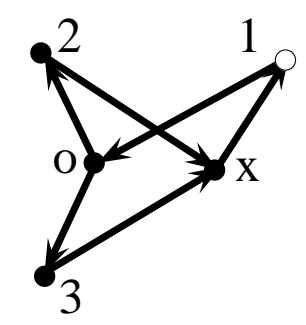

Figure 76

Suppose $x$ is connected to exactly two of the nodes $\mathbf{1 , 2 , 3}$. There are three cases:

1. Suppose $x$ is connected to nodes $\mathbf{1 , 2} . x$ can only be contained in the neighborhoods as shown in picture 4 or 5 in Figure 74 . Edges $a, b$ comes from the same triangle. Similarly, if $x$ is connected to nodes $\mathbf{1 , 3}$, edges $a, c$ are in the same triangle.

2. Suppose $x$ is connected to nodes $\mathbf{2 , 3}$. None of the pictures in Figure 74 contains such a neighborhood. Hence the graph is indecomposable.

Assume $x$ is connected to exactly one of the nodes $\mathbf{1 , 2 , 3}$. First, suppose $x$ is connected to node $\mathbf{1}$ which is an endpoint of an inward edge. Note that nodes $\mathbf{2}$ and $\mathbf{3}$ can not be connected to node $\mathbf{1}$. Use the argument in the previous section. If the graph is decomposable, we conclude the following:

- If nodes $\mathbf{1 , 2}$ are connected, edges $a, b$ come from the same triangle and edge $c$ comes from a spike.

- If nodes $\mathbf{1 , 3}$ are connected, edges $a, c$ come from the same triangle and edge $b$ comes from a spike.

- If nodes $\mathbf{2 , 3}$ are both disconnected from node $\mathbf{1}$, edges $b, c$ come from the same fork and edge $a$ comes from a spike.

Suppose $x$ is connected to node $\mathbf{2}$. If the graph is decomposable and node $\mathbf{1 , 2}$ are connected, then $a, b$ come from the same triangle. If nodes $\mathbf{1 , 2}$ are disconnected, then $a, c$ come from the same triangle. The criterion is similar if $x$ is connected only to node $\mathbf{3}$.

Next, suppose $n=2$, and denote these two corresponding nodes by $x, y$.

First, check if they are both connected to node $\mathbf{1}$. If it is this case, neither node $\mathbf{2}$ or $\mathbf{3}$ can be connected to node $\mathbf{1}$. Moreover, according to the argument in the previous section, we have two cases: (1). a comes from a spike and $b, c$ come from a fork; (2). $a$ comes from a triangle. In the second case, $x, y$ must both be connected to nodes $\mathbf{2}$ or $\mathbf{3}$ by edges with compatible directions, and edges $a, b$ (edges $a, c$ ) are in the same triangle, see Figure 74, picture 5 .

If $x, y$ are not both connected to node $\mathbf{1}$, check if they are both connected to node $\mathbf{2}$. If so, edges $a, c$ can only be obtained from a triangle and $b$ comes from a spike. Since $n=2$, there is no node other than $o$ that is connected to node $\mathbf{1}$ or $\mathbf{3}$. Therefore, the neighborhood is as in Figure 77. Note that node $o$ is contained in a neighborhood listed in picture 14 Figure 74 . The argument is similar if $x, y$ are both connected to node 3 . 


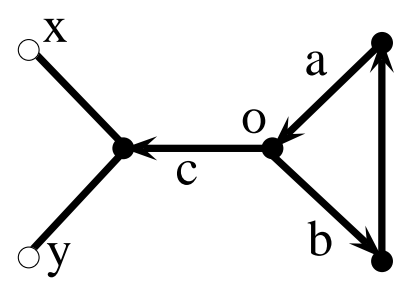

Figure 77

Next suppose $x, y$ are not connected to the same node. If the graph is decomposable, there are the following cases:

- $x$ is only connected to node $\mathbf{1}$ and $y$ only to node $\mathbf{2}$. In this case, nodes $\mathbf{1 , 2}$ must be connected and $a, b$ come from the same triangle. It's similar if $x$ is only connected to node $\mathbf{1}$ and $y$ only to node $\mathbf{3}$.

- $x$ is only connected to nodes $\mathbf{1 , 2}$ and $y$ only to node $\mathbf{3}$. In this case, $a, b$ come from the same triangle. If nodes $\mathbf{1 , 2}$ are connected, node $o$ is contained in a neighborhood as shown in Figure 74, picture 13. If nodes $\mathbf{1 , 2}$ are disconnected, the neighborhood is as shown in picture 8 (node $p=x$ ).

- $x, y$ are connected to nodes $\mathbf{2 , 3}$ respectively. In addition, if nodes $\mathbf{1 , 2}$ are connected, then edges $a, b$ come from the same triangle. The neighborhood is as shown in picture 11. Similarly, if nodes $\mathbf{1 , 3}$ are connected, edges $a, c$ come from the same triangle. Notice that in this case, nodes $\mathbf{2}$ and $\mathbf{3}$ can not be connected to node $\mathbf{1}$ at the same time, neither can they both be disconnected at the same time.

Last of all, suppose $n=3$. Denote the three corresponding nodes by $x, y, z$. According to the argument in the previous section, in this case, the graph $G$ is decomposable only if node $\mathbf{1}$ is connected to node $\mathbf{2}$ or $\mathbf{3}$, forming a triangle with the corresponding edges. See Figure 71.

Theorem 1. Assume $G$ is a connected quiver such that every node in $G$ has degree less than or equal to three. If each of the nodes of degree three has a neighborhood as in one of the pictures listed in Figure 74 (up to a change of orientations of all edges), then $G$ is decomposable. Otherwise, $G$ is indecomposable.

Proof. If $G$ is either the graph in pictures 2 or the one in 6 in Figure 74, the result is evident. Suppose $G$ is neither the graph in picture 2 nor the one in picture 6 . Apply replacements to corresponding neighborhoods in Figure 74 . Denote the new graph by $G^{\prime}$. Notice that according to Lemma 1 , if $G^{\prime}$ is decomposable, so is $G$. The replacements for all neighborhoods in Figure 74 contain triangular blocks. Each node of degree three in $G^{\prime}$ has a neighborhood in the form of Figure 62 and is obtained from gluing a triangular block to a spike. Remove all the triangles and the resulting graph is a disconnected union of paths. Note that since all forks have been replaced in the previous steps, all the paths are obtained by gluing of finitely many spikes in a unique way. Since the new graph has a unique decomposition, so does the original graph. 
As an application of the algorithm we can classify all decomposable graphs with nonunique decompositions:

Theorem 2. Any connected decomposable graph $G$ with non-unique decompositions is one of the graphs from Figure 78, up to the change of orientations of all edges in $G$.
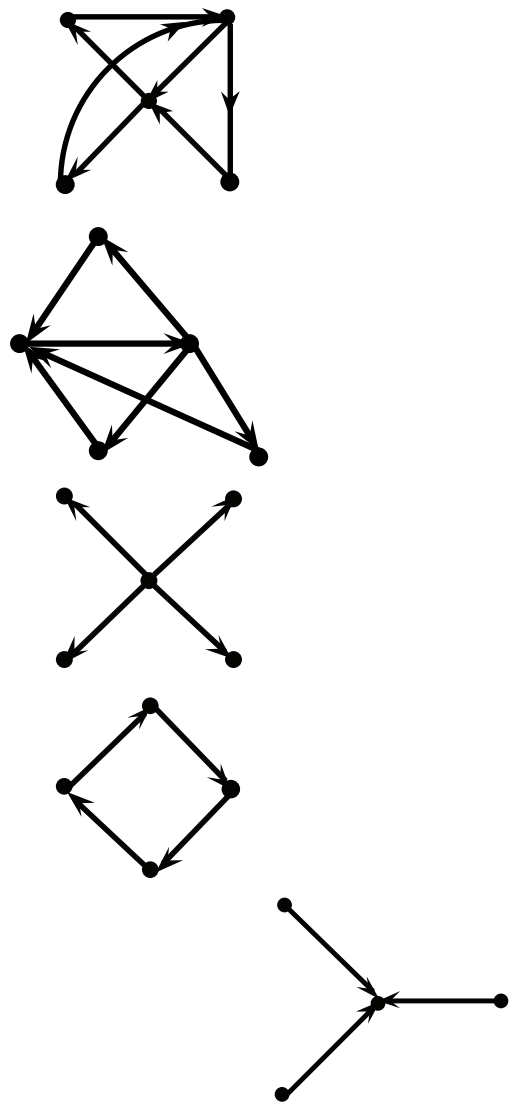
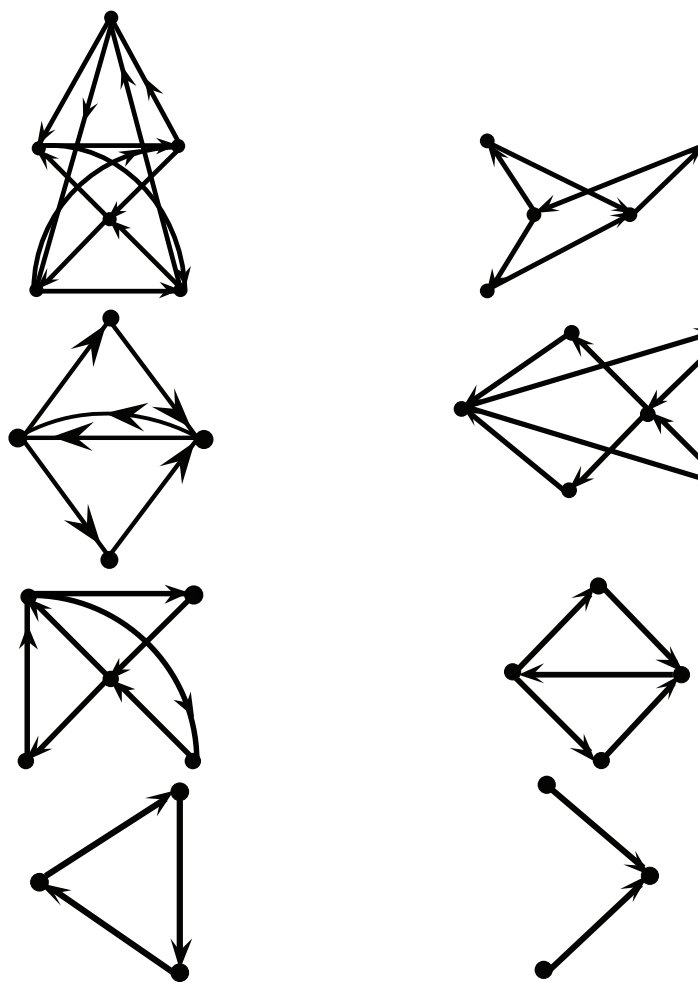

Figure 78: Neighborhoods that Have Non-unique Decomposition

Corollary 2. Suppose $G$ is a finite quiver with non-unique decompositions. If $G$ does not contain isolated nodes, then it only has finitely many possible decompositions.

Sketch of Proof: Recall that a disconnected decomposable graph $\widetilde{G}$ can not be obtained from gluing a block to a connected decomposable graph unless $\widetilde{G}$ contains isolated nodes. If a finite graph $G$ has infinitely many different decompositions, it would mean that there are infinitely many decompositions such that the edges in a large portion (not just one edge) are annihilated and remaining part coincides with graph $G$. This means that $G$ contains isolated nodes in the portion where all edges are annihilated.

Lemma 9. The decomposition algorithm is linear in the number of nodes in the graph. 
Proof. At each stage of simplification, the number of replacements that we need to apply is no more than the number of nodes in the graph. Moreover, if the replacement is applied to a neighborhood of a node, the degree of the considered node becomes three. Thus a replacement reduces the degree of the considered node to three if it is larger than three in the original graph. Moreover, any node of degree three in the replacement is obtained by gluing a triangle to a spike, so we do not apply replacement again in the stage when we consider nodes of degree three. This means replacement is applied at most once to the same node. To conclude, the number of replacements is less than the number of nodes in the graph.

In [2], Fomin, Shapiro and Thurston define an operation on quivers called mutation. A quiver is said to have finite mutation type if its mutation class is finite. It was noticed by P.Tumarkin that our algorithm provides a fast way to determine when a quiver of size larger than 10 has finite mutation type (see [1] for details).

Theorem 3. There exists an algorithm which is linear in number of nodes to check if any quiver has finite mutation type.

Proof. By the proposition in Section 2, we can assume that the given quiver $G$ is connected. In [1], the author proved that a quiver with finite mutation type is either decomposable or one of the 11 exceptional types whose size does not exceed 10 (see Theorem 6.1 in [1]). We apply our algorithm to check if $G$ is decomposable. By the previous lemma, the number of operations is linear in the number of nodes in $G$. If the algorithm shows that $G$ is indecomposable, and the size of $G$ does not exceed 10, we further check if $G$ coincides with one of the 11 exceptional types in Theorem 6.1 [1]. The last step takes only finite number of operations, this finishes the algorithm.

\section{Acknowledgement}

I thank P. Tumarkin for the discovery of the linearity of this algorithm, and S. Fomin, M. Shapiro and D. Thurston for bringing up this problem in [2]. I especially thank my ad-

visor Dr. Shapiro for helpful advice and inspiring discussions, and for kindly proofreading this paper.

\section{References}

[1] A. Felikson, M. Shapiro, and P. Tumarkin. Skew-symmetric cluster algebras of finite mutation type. arXiv:0811.1703.

[2] S. Fomin, M. Shapiro, and D. Thurston. Cluster algebras and triangulated surfaces I: Cluster complexes. Acta Mathematica, 201, October 2008. 